\title{
A New Distance-Based Consensus Reaching Model for Multi-Attribute Group Decision-Making with Linguistic Distribution Assessments
}

\author{
Shengbao Yao* \\ School of Business Administration, Zhongnan University of Economics and Law, Wuhan, Hubei 430073, China
}

ARTICLE IN F O
Article History
Received 25 Nov 2018
Accepted 04 Feb 2019
Keywords
Multi-attribute group
decision-making
Linguistic distribution assessments
Consensus reaching
Distance measure
Optimization

\section{INTRODUCTION}

The increasing complexity of the social and economic environment nowadays makes it more and more impracticable for a single decision maker or expert to consider all the relevant factors of a decision-making problem [1]. For this reason, many companies, organizations, and administrations employ multiple members in complex decision-making processes, which is known as group decision-making (GDM). Generally, GDM can be understood as a process to aggregate individual opinions of multiple experts so as to acquire a group opinion in the situations where experts verbalize their preferences regarding multiple alternatives [2].

Many GDM problems present quantitative aspects which can be evaluated by means of exact numerical values. However, some problems present also qualitative features that are complex to assess by using precise numerical values. In this circumstances, experts usually articulate their preferences with qualitative information that are represented by linguistic variables. In the classical linguistic computational models [3, 4], experts are restricted to express their opinions with a single linguistic term. In complex decision problem under uncertainty, experts may hesitate between different linguistic terms and require richer expressions to express their preferences more accurately. For this reason, Rodríguez et al. [5] proposed the concept of hesitant fuzzy linguistic term set (HFLTS), which is

*Corresponding author. Email: yaoshengbao@hotmail.com used to enhance the flexibility and richness of linguistic elicitation by experts in hesitant situations under qualitative settings [6]. To provide more flexible and richer linguistic expressions, researchers recently have proposed several distribution-based hesitant fuzzy linguistic information such as linguistic distribution assessments [7], possibility distribution for HFLTS [8], proportional HFLTS [9], probabilistic linguistic term set $[10,11]$, probability-based interpretation for the linguistic expressions [12]. All these distributionbased hesitant fuzzy linguistic information have successfully served to solve decision-making problems for their specified backgrounds. Among these distribution-based hesitant fuzzy linguistic information types, linguistic distribution assessment has received increasing attention from many scholars [13-15]. Our interest in this paper is focused on GDM problem with information represented by means of linguistic distribution assessments.

In GDM, a group of experts initially may have very different opinions due to the different background, knowledge, and experience of experts. Therefore, it is necessary to develop a consensus reaching process (CRP) to help experts achieve agreement. Traditionally, the ideal consensus can be understood as a full and unanimous agreement of all experts preferences with respect to all the feasible alternatives. This type of consensus is an utopian consensus and it is very difficult to achieve in real-world GDM problems, which has led to the use of a new concept called "soft" consensus level [16]. Under the soft consensus framework, the CRP can be modelled as an iterative group discussion process coordinated by a moderator, who 
helps the experts to make their preferences closer [17]. To date, the CRP based on the "soft" consensus have attracted wide attentions from researchers in the field of GDM [17-23]. In the process of GDM in complex environment, experts may express their preferences by using preference relations (PRs) with fuzzy linguistic information [18]. Accordingly, a variety of consensus models have been developed for GDM problems in which the preferences are represented by means of PRs with fuzzy linguistic information $[7,17,18,24-37]$. In some GDM situations, the decision information regarding the alternatives is represented in the form of multi-attribute decision matrix. Similarly, when solving the multiattribute GDM (MAGDM) problems, the CRP is still necessary to reach an agreement among experts before making a common decision. For MAGDM problems in linguistic information environment, authors have also proposed a number of consensus models. For example, Xu et al. [38] developed an interactive consensus model for MAGDM problems with uncertain linguistic information that is evaluated in different unbalanced linguistic label sets. For MAGDM with multi-granular linguistic term sets, Parreiras et al. [39] introduced a flexible consensus scheme which allows one to rationally aggregate the preferences of a group of experts into a consistent collective preference. Later, Rosello et al. [40] proposed a methodology into MAGDM by using consensus within multi-granular linguistic assessments. In order to solve MAGDM problems under an uncertain linguistic environment, Xu et al. [41] proposed a CRP model to help experts reach a satisfactory consensus. From the perspective of social network analysis, Wu et al. [42] introduced a framework to preference estimation and consensus building for multiple criteria GDM with incomplete linguistic information. Sun et al. [43] proposed a new approach to consensus measurement of MAGDM with linguistic PRs. For 2-tuple linguistic MAGDM with incomplete weight information, Zhang et al. [44] presented a consensus reaching model in which a weight-updating model is employed to derive the weights to eliminate the conflict in the group. For MAGDM under uncertain linguistic environment, Pang et al. [45] developed an interactive consensus model with adaptive experts weights. Recently, Zhang et al. [46] proposed a distance-based consensus measure and presented a minimum adjustment distance consensus rule for MAGDM with HFLTS. By using the relative projection model, Zhang et al. [47] proposed a consensus model for MAGDM with hesitant linguistic information on multi-granular linguistic term sets.

Through the above analysis of the relevant literatures, we can see that previous studies have made significant contributions to the consensus models of GDM problems with fuzzy linguistic information. However, a more detailed survey of the literature showed that consensus modeling of MADGM problem with linguistic distribution assessment has not been adequately considered. Therefore, this paper focuses on GDM problems in which the experts express their preferences by means of multi-attribute decision matrix with linguistic distribution assessments. Comparing with the existing consensus models for MAGDM problems, our contributions can be mainly summarized in the following aspects:

1. As we know, distance and similarity measures play an important role in developing effective CRP model. To study the applications of linguistic distribution assessments, several distance measures between linguistic distribution assessment have been proposed $[7,14,15]$. However, as discussed in Section 3, the existing distance measures are not free from drawbacks. In order to overcome the limitations of the existing distance measures, we propose a novel distance measure for linguistic distribution assessments based on the concept of cumulative proportion distribution. We also find that the proposed distance measure satisfies several desirable properties.

2. Popular consensus models for MAGDM problems can be divided into the interactive ones and the automatic ones [45]. Generally, the interactive consensus models are usually lack of effective feedback mechanisms to guide the experts to make quick adjustments, while the automatic ones cannot reflect the subjective adjustment opinions from the experts during the CRP. For MAGDM problem with linguistic distribution assessment, a novel consensus reaching model is developed in this paper. The proposed consensus reaching model not only can reflect experts additional preferences during the CRP but also can automatically generate advices for preference adjustment.

3. One of the most significant issues in the consensus reaching model is how to design an effective feedback mechanism to guide experts reach consensus with minimum adjustments. A hybrid feedback mechanism is designed by combining an identification rule (IR) and an optimization based model in the proposed consensus reaching model. During CRP, an optimization model is solved in each iteration to minimize the the deviation between the adjusted values and initial preferences, which in turn leads to the good performance of the proposed consensus reaching model in preserving the initial preference information.

The rest of the paper is organized as follows: Section 2 summaries the basic concepts of the 2-tuple linguistic representation model and linguistic distribution assessment. In Section 3, we analyze the existing distance measures, and point out their drawbacks with numerical counterexamples. In addition, a novel distance measure is defined for linguistic distribution assessments. Section 4 establishes a consensus support model for MAGDM problems under linguistic distribution assessment environment. Numerical examples and a comparison study are presented in Section 5 to illustrate the performance of the proposed method. Finally, we conclude the paper in Section 6.

\section{PRELIMINARIES}

This section reviews some relevant knowledge regarding the 2-tuple linguistic representation model and the concept of linguistic distribution assessments.

\subsection{Linguistic Information and 2-Tuple Linguistic Representation Model}

Suppose that $S=\left\{s_{0}, s_{1}, \ldots, s_{g-1}\right\}$ is a linguistic term set with odd cardinality $g$, such as 5 or 7 , where each label $s_{i}$ represents a possible value of a linguistic variable. Usually, it requires that the linguistic term set $S$ satisfies the following characteristics: 1 ) the set of the linguistic terms is ordered: $s_{i} \geq s_{j}$ if $\left.i \geq j ; 2\right)$ there exists a negation operator "Neg" such that $\left.\operatorname{Neg}\left(s_{i}\right)=s_{g-i} ; 3\right)$ the maximization operator " $\max$ ": $\max \left(s_{i}, s_{j}\right)=s_{i}$ if $s_{i} \geq s_{j}$; and 4) the minimization operator " $\min$ ": $\min \left(s_{i}, s_{j}\right)=s_{i}$ if $s_{i} \leq s_{j}$. 
In order to avoid information loss in computing with words, Herrera and Martínez [4] introduced the 2-tuple linguistic representation model on the basis of the symbolic translation. In this model, a 2 -tuple $\left(s_{i}, \alpha_{i}\right)$ is used to represent linguistic information, where $s_{i}$ is a linguistic term pertaining to the predefined linguistic term set and $\alpha_{i} \in[-0.5,0.5)$ represents the symbolic translation. Specifically, the 2-tuple linguistic representation model is formally defined as follows:

Definition 2.1. ([4]). Let $S=\left\{s_{0}, s_{1}, \ldots, s_{g}\right\}$ be a linguistic term set and $\beta \in[0, g]$ be a value representing the result of a symbolic aggregation operation, then the 2 -tuple that expresses the equivalent information to $\beta$ is obtained with the following function:

$$
\begin{aligned}
& \Delta:[0, g] \mapsto S \times[-0.5,0.5) \\
& \Delta(\beta)=\left(s_{i}, \alpha_{i}\right)
\end{aligned}
$$

where $i=\operatorname{round}(\beta)$ and $\alpha_{i}=\beta-i$, where "round" is the usual rounding operator, $s_{i}$ has the closest index label to $\beta$, and $\alpha_{i}$ is the value of the symbolic translation.

According the above definition, a linguistic term $s_{i}$ belonging to the linguistic term set $S$ can be regarded as a 2-tuple linguistic by adding a value 0 to it as symbolic translation. That is, $s_{i} \in S \Rightarrow\left(s_{i}, 0\right)$. For convenience's sake, we will use 2-tuple linguistic representations instead of linguistic terms in the following.

Definition 2.2. ([4]). Let $S=\left\{s_{0}, s_{1}, \ldots, s_{g}\right\}$ be a linguistic term set and $\left(s_{i}, \alpha_{i}\right)$ be a 2 -tuple, there exists a function

$$
\begin{aligned}
& \Delta^{-1}: S \times[-0.5,0.5) \mapsto[0, g] \\
& \Delta^{-1}\left(\left(s_{i}, \alpha_{i}\right)\right)=i+\alpha_{i}=\beta
\end{aligned}
$$

that uniquely transforms a 2 -tuple into its equivalent numerical value $\beta \in[0, g]$.

Definition 2.3. ([4]). Let $x=\left\{\left(s_{1}, \alpha_{1}\right),\left(s_{2}, \alpha_{2}\right), \cdots,\left(s_{n}, \alpha_{n}\right)\right\}$ be a set of 2-tuples and $W=\left\{w_{1}, \cdots, w_{n}\right\}$ be their associated weights. The 2-tuple weighted average $\bar{x}$ is defined as

$$
\bar{x}=\Delta\left(\frac{\sum_{i=1}^{n} \Delta^{-1}\left(s_{i}, \alpha_{i}\right) \cdot w_{i}}{\sum_{i=1}^{n} w_{i}}\right) .
$$

\subsection{Linguistic Distribution Assessments}

In [7], Zhang et al. defined the concept of distribution assessments in a linguistic term set. In this subsection, we review some related knowledge regarding linguistic distribution assessment.

Definition 2.4. [7] Let $S=\left\{s_{0}, s_{1}, \ldots, s_{g-1}\right\}$ be a linguistic term set. Let $m=\left\{\left(s_{k}, \beta_{k}\right) \mid k=0,1, \ldots, g-1\right\}$, where $s_{k} \in S, \beta_{k} \geq 0, \sum_{k=0}^{g-1} \beta_{k}=$ 1 and $\beta_{k}$ is the symbolic proportion of $s_{k}$, then $m$ is called a distribution assessment of $S$.
Definition 2.5. [7] Let $m=\left\{\left(s_{k}, \beta_{k}\right) \mid k=0,1, \ldots, g-1\right\}$, where $s_{k} \in S, \beta_{k} \geq 0, \sum_{k=0}^{g-1} \beta_{k}=1$ be a distribution assessment of $S$, the expectation of $m$ is defined as a linguistic 2-tuple $E(m)$ :

$$
E(m)=\triangle\left(\sum_{k=0}^{g-1} k \beta_{k}\right)
$$

Zhang et al. [7] developed the following computational model for the distribution assessment of $S$. Let $m_{1}$ and $m_{2}$ be two distribution assessments of $S$. Then

1. A comparison operator:

i. if $E\left(m_{1}\right)<E\left(m_{2}\right)$, then $m_{1}$ is smaller than $m_{2}$;

ii. if $E\left(m_{1}\right)=E\left(m_{2}\right)$, then $m_{1}$ and $m_{2}$ have the same expectation.

2. A weighted averaging operator of linguistic distribution assessments are defined as follows:

Definition 2.6. [7] Let $\left\{m_{1}, m_{2}, \ldots, m_{n}\right\}$ be a set of linguistic distribution assessments of $S$, where $m_{i}=\left\{\left(s_{k}, \beta_{k}^{i}\right) \mid k=0,1, \ldots, g-1\right\}$, $i=1,2, \ldots, n$ and $w=\left(w_{1}, w_{2}, \ldots, w_{n}\right)^{T}$ be an associated weighting vector that satisfies $w_{i} \geq 0$ and $\sum_{i=1}^{n} w_{i}=1$, then the weighted averaging operator of $\left\{m_{1}, m_{2}, \ldots m_{n}\right\}$ is defined as

$$
D A W A_{w}\left\{m_{1}, m_{2}, \ldots, m_{n}\right\}=\left\{\left(s_{k}, \beta_{k}\right) \mid k=0,1, \ldots, g-1\right\},
$$

where $\beta_{k}=\sum_{i=1}^{n} w_{i} \beta_{k}^{i}, k=0,1, \ldots, g-1$.

\section{AN IMPROVED DISTANCE MEASURE FOR LINGUISTIC DISTRIBUTION ASSESSMENTS}

Distance and similarity measures are common tools used widely in measuring the deviation and proximity degrees of different arguments [48]. To study the applications of linguistic distribution assessments, several distance measures between linguistic distribution assessments have been proposed. In this section, we point out some drawbacks of the existing distance measures by counterexamples. Furthermore, we introduce a new distance and similarity measure between linguistic distribution assessments to overcome such drawbacks.

Zhang et al. [7] defined the distance between linguistic distribution assessments $m_{1}$ and $m_{2}$ as follows:

Definition 3.1. [7]: Let $m_{1}=\left\{\left(s_{k}, \beta_{k}^{1}\right) \mid k=0,1, \ldots, g-1\right\}$ and $m_{2}=\left\{\left(s_{k}, \beta_{k}^{2}\right) \mid k=0,1, \ldots, g-1\right\}$ be two linguistic distribution assessments of a linguistic term set $S$, then the distance between $m_{1}$ and $m_{2}$ is defined as

$$
d_{1}\left(m_{1}, m_{2}\right)=\frac{1}{2} \sum_{k=0}^{g-1}\left|\beta_{k}^{1}-\beta_{k}^{2}\right| .
$$

The drawback of the distance measure defined by Eq. (4) is shown with Example 1. 
Example 1. Let $S^{\text {example }}=\left\{s_{0}, s_{1}, s_{2}, s_{3}, s_{4}, s_{5}, s_{6}\right\}$ be a linguistic term set and there are three linguistic distribution assessments: $m_{1}=\left\{\left(s_{0}, 0.5\right),\left(s_{1}, 0.5\right),\left(s_{2}, 0\right),\left(s_{3}, 0\right),\left(s_{4}, 0\right),\left(s_{5}, 0\right),\left(s_{6}, 0\right)\right\}$, $m_{2}=\left\{\left(s_{0}, 0\right),\left(s_{1}, 0\right),\left(s_{2}, 0\right),\left(s_{3}, 0.5\right),\left(s_{4}, 0.5\right),\left(s_{5}, 0\right),\left(s_{6}, 0\right)\right\}$, $m_{3}=\left\{\left(s_{0}, 0\right),\left(s_{1}, 0\right),\left(s_{2}, 0\right),\left(s_{3}, 0\right),\left(s_{4}, 0\right),\left(s_{5}, 0.5\right),\left(s_{6}, 0.5\right)\right\}$. By Definition 3.1, we can obtain $d\left(m_{1}, m_{2}\right)=$ $\frac{1}{2}(0.5+0.5+0+0.5+0.5+0+0)=1$ and $d\left(m_{1}, m_{3}\right)=$ $\frac{1}{2}(0.5+0.5+0+0+0+0.5+0.5)=1$ which means that the distance between $m_{1}$ and $m_{2}$ is equal to that between $m_{1}$ and $m_{3}$. Obviously it is unreasonable.

Zhang et al. [14] pointed out that the distance measure defined by Eq. (4) just calculates the deviation between symbolic proportions and ignores the linguistic terms. To overcome the drawback, Zhang et al. [14] proposed a new distance measure as follows:

Definition 3.2. [14]: Let $m_{1}=\left\{\left(s_{k}, \beta_{k}^{1}\right) \mid k=0,1, \ldots, g-1\right\}$ and $m_{2}=\left\{\left(s_{k}, \beta_{k}^{2}\right) \mid k=0,1, \ldots, g-1\right\}$ be two linguistic distribution assessments of a linguistic term set $S$, then the distance between $m_{1}$ and $m_{2}$ is defined as

$$
d_{2}\left(m_{1}, m_{2}\right)=\frac{1}{g-1}\left|\sum_{k=0}^{g-1}\left(\beta_{k}^{1}-\beta_{k}^{2}\right) k\right|
$$

Reconsider Example 1. By Definition 3.2, we can obtain $d\left(m_{1}, m_{2}\right)=0.50$ and $d\left(m_{1}, m_{3}\right)=0.83$. Indeed, the distance measure defined by Eq. (5) overcomes the limitation of the distance measure defined by Eq. (4) to a certain extent. However, the distance measure defined by Eq. (5) also has limitation, which can be illustrated by the following Example 2:

Example 2. Let $S^{\text {example }}=\left\{s_{0}, s_{1}, s_{2}, s_{3}, s_{4}, s_{5}, s_{6}\right\}$ be a linguistic term set and there are three linguistic distribution assessments: $m_{1}=\left\{\left(s_{0}, 0\right),\left(s_{1}, 0\right),\left(s_{2}, 0.1\right),\left(s_{3}, 0.8\right),\left(s_{4}, 0.1\right),\left(s_{5}, 0\right),\left(s_{6}, 0\right)\right\}$, $m_{2}=\left\{\left(s_{0}, 0\right),\left(s_{1}, 0.1\right),\left(s_{2}, 0.2\right),\left(s_{3}, 0.4\right),\left(s_{4}, 0.2\right),\left(s_{5}, 0.1\right),\left(s_{6}, 0\right)\right\}$. Obviously, we can see that $m_{1}$ and $m_{2}$ are two different linguistic distribution assessments, that is, $m_{1} \neq m_{2}$. However, by definition 3.2 , we obtain $d\left(m_{1}, m_{2}\right)=0$. According to definition 3.2 , it is not difficult to infer that $d\left(m_{1}, m_{2}\right)=0$ if $E\left(m_{1}\right)=E\left(m_{2}\right)$. We argue that an ideal distance measure for linguistic distribution assessment should possess the basic property: $d\left(m_{1}, m_{2}\right)=0$ if and only if $m_{1}=m_{2}$.

Recently, Yu et al. [15] defined a new distance measure between linguistic distribution assessments as follows:

Definition 3.3. [15]: Let $m_{1}=\left\{\left(s_{1}^{k_{1}}, \beta_{1}^{k_{1}}\right) \mid k_{1}=1,2, \ldots, \sharp m_{1}\right\}$ and $m_{2}=\left\{\left(s_{2}^{k_{2}}, \beta_{2}^{k_{2}}\right) \mid k_{1}=1,2, \ldots, \sharp m_{2}\right\}$ be two linguistic distribution assessments of a linguistic term set $S$, where $\sharp m_{i}$ is the number of linguistic terms in $m_{i}, \beta_{i}^{k_{i}}>0$ and $\sum_{k_{i}=1}^{\sharp m_{i}} \beta_{i}^{k_{i}}=1, i=1,2$. The generalized distance measure between two linguistic distribution assessments $m_{1}$ and $m_{2}$ can be defined by Eq. (6), where $f$ is the linguistic scale function presented in Yu et al. [15] and $r>0$.

The advantage of the distance measure defined by Eq. (6) can measure the distance between some unequal linguistic distribution assessments which previous distance measurement cannot measure [15]. However, it is not free from drawbacks. $d_{g}\left(m_{1}, m_{2}\right)$
$=\left[\frac{1}{2}\left(\frac{1}{\sharp m_{1}} \sum_{\left(s_{1}^{k_{1}}, \beta_{1}^{k_{1}}\right) \in m_{1}} \min _{\left(s_{2}^{k_{2}}, \beta_{2}^{k_{2}}\right) \in m_{2}}\left(\left|f\left(s_{1}^{k_{1}}\right) \beta_{1}^{k_{1}}-f\left(s_{2}^{k_{2}}\right) \beta_{2}^{k_{2}}\right|\right)^{r}\right.\right.$
$\left.\left.+\frac{1}{\sharp m_{2}} \sum_{\left(s_{2}^{k_{2}}, \beta_{2}^{k_{2}}\right) \in m_{2}} \min _{\left(s_{1}^{k_{1}}, \beta_{1}^{k_{1}}\right) \in m_{1}}\left(\left|f\left(s_{1}^{k_{1}}\right) \beta_{1}^{k_{1}}-f\left(s_{2}^{k_{2}}\right) \beta_{2}^{k_{2}}\right|\right)^{r}\right)\right]^{\frac{1}{r}}$,

Example 3. Let $S^{\text {example }}=\left\{s_{0}, s_{1}, s_{2}, s_{3}, s_{4}, s_{5}, s_{6}\right\}$ be a linguistic term set and there are three linguistic distribution assessments: $m_{1}=\left\{\left(s_{0}, 0\right),\left(s_{1}, 0\right),\left(s_{2}, 0\right),\left(s_{3}, 0\right),\left(s_{4}, 0\right),\left(s_{5}, 0.2\right),\left(s_{6}, 0.8\right)\right\}$, $m_{2}=\left\{\left(s_{0}, 0\right),\left(s_{1}, 0\right),\left(s_{2}, 0.5\right),\left(s_{3}, 0.5\right),\left(s_{4}, 0\right),\left(s_{5}, 0\right),\left(s_{6}, 0\right)\right\}$, $m_{3}=\left\{\left(s_{0}, 0\right),\left(s_{1}, 0\right),\left(s_{2}, 0\right),\left(s_{3}, 0.5\right),\left(s_{4}, 0.5\right),\left(s_{5}, 0\right),\left(s_{6}, 0\right)\right\}$. By definition $3.3(r=1)$, we can obtain $d\left(m_{1}, m_{2}\right)=$ $\frac{1}{2}\left(\frac{1}{2}(0+0.55)+\frac{1}{2}(0+0.0833)\right)=0.1583$ and $d\left(m_{1}, m_{3}\right)=$ $\frac{1}{2}\left(\frac{1}{2}(0.0833+0.4667)+\frac{1}{2}(0.0833+0.1667)\right)=0.2000$ which means that the distance between $m_{1}$ and $m_{2}$ is smaller to that between $m_{1}$ and $m_{3}$. Obviously it is unreasonable.

Based on the above analysis, this paper attempts to develop a new distance measure between distribution assessments by making full use of the proportion distribution information of linguistic distribution assessment. Similar to the cumulative probability distribution, the concept of cumulative proportion distribution can be derived for linguistic distribution assessment. In particular, the vector $\left(\sum_{r=0}^{0} \beta_{r}, \sum_{r=0}^{1} \beta_{r}, \ldots, \sum_{r=0}^{k} \beta_{r}, \ldots, \sum_{r=0}^{g-1} \beta_{r}\right)$ is defined as the cumulative proportion distribution of linguistic distribution assessment $m=\left\{\left(s_{k}, \beta_{k}\right) \mid k=0,1, \ldots, g-1\right\}$. Using the cumulative proportion distribution, we can define a distance measure between linguistic distribution assessments $m_{1}$ and $m_{2}$ by computing their difference in cumulative proportion distributions. The proposed distance measure is given below.

Definition 3.4. Let $m_{1}=\left\{\left(s_{k}, \beta_{k}^{1}\right) \mid k=0,1, \ldots, g-1\right\}$ and $m_{2}=$ $\left\{\left(s_{k}, \beta_{k}^{2}\right) \mid k=0,1, \ldots, g-1\right\}$ be two linguistic distribution assessments of a linguistic term set $S$, then the distance between two linguistic distribution assessments $m_{1}$ and $m_{2}$ is defined as

$$
d\left(m_{1}, m_{2}\right)=\frac{1}{g-1} \sum_{k=0}^{g-1}\left|\sum_{r=0}^{k} \beta_{r}^{1}-\sum_{r=0}^{k} \beta_{r}^{2}\right|
$$

Example 4. Reconsider the linguistic distribution assessments in Example 2: $m_{1}=\left\{\left(s_{0}, 0\right),\left(s_{1}, 0\right),\left(s_{2}, 0.1\right),\left(s_{3}, 0.8\right),\left(s_{4}, 0.1\right),\left(s_{5}, 0\right)\right.$, $\left.\left(s_{6}, 0\right)\right\}, m_{2}=\left\{\left(s_{0}, 0\right),\left(s_{1}, 0.1\right),\left(s_{2}, 0.2\right),\left(s_{3}, 0.4\right),\left(s_{4}, 0.2\right),\left(s_{5}, 0.1\right)\right.$, $\left.\left(s_{6}, 0\right)\right\}$. For $m_{1}$ and $m_{2}$, we can calculate their cumulative proportion distributions $C P_{1}=(0,0,0.1,0.9,1,1,1)$ and $\mathrm{CP}_{2}=(0,0.1,0.3,0.7,0.9,1,1)$. By using Eq. (7), we have $d\left(m_{1}, m_{2}\right)=0.1$. It is worth noting that the proposed distance measure defined by Eq. (7) has very clear geometric meaning. Figure 1 shows the proportion distribution of linguistic distribution assessments $m_{1}$ and $m_{2}$ (for convenience, the subscripts of linguistic term are taken as the values of variable). The distance 


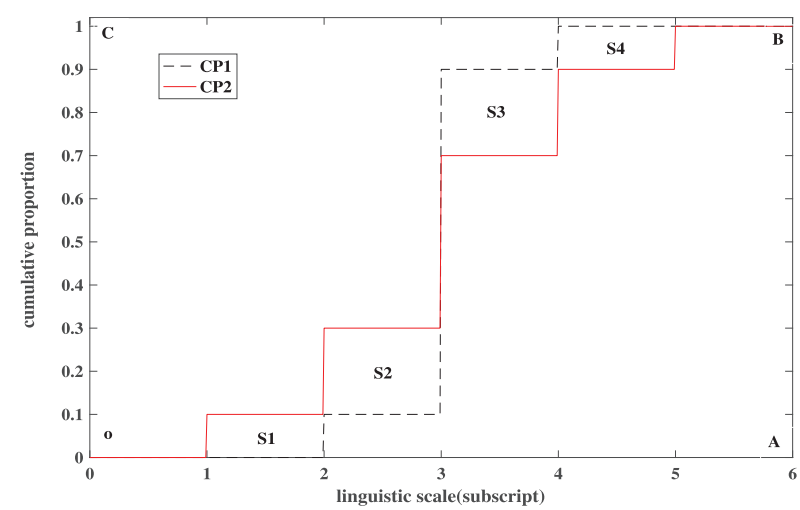

Figure 1 The geometric interpretation of the proposed distance measure.

$d\left(m_{1}, m_{2}\right)$ between two linguistic distribution assessments $m_{1}$ and $m_{2}$ can be explained as the ratio of intersecting area of the corresponding cumulative proportion distributions and $g-1$. From Figure 1 , we can see that the distance $d\left(m_{1}, m_{2}\right)$ equals the ratio of the sum of area of $S 1, S 2, S 3, S 4$, and the area of rectangle OABC. Clearly, the larger the intersecting area between the two cumulative proportion distributions is, the farther the distance between the two linguistic distribution assessments is.

In order to analyze the performance of the proposed distance measure, we conduct a comparative analysis by using numerical examples. Reconsider Example 1, Example 2, and Example 3. We calculate the values of the distance by applying the proposed distance measure defined by Equation (7) and the existing distance measures defined by Equations. (4-6). Table 1 shows the comparison results. As we can see from Table 1 on the one hand, the existing distance measures show some limitations, which has been explained in detail in section 3. On the other hand, we can also see that the proposed distance measure in this paper not only can distinguish linguistic distribution assessments as shown in Example 1 , in which the positive proportions are distributed on different linguistic terms, but also can distinguish linguistic distribution assessments with the same expectation values. Moreover, for the linguistic distribution assessments in Example 3, the results calculated by the proposed distance measure are more reasonable than that calculated by the distance measure proposed in [15]. From above analysis, we can conclude that the proposed distance measure for linguistic distribution assessments exhibits better performance.

For the proposed distance measure, we can obtain the following properties.
Theorem 3.1. Let $m_{1}=\left\{\left(s_{k}, \beta_{k}^{1}\right) \mid k=0,1, \ldots, g-1\right\}, m_{2}=$ $\left\{\left(s_{k}, \beta_{k}^{2}\right) \mid k=0,1, \ldots, g-1\right\}$ and $m_{3}=\left\{\left(s_{k}, \beta_{k}^{3}\right)=\mid k=\right.$ $0,1, \ldots, g-1\}$ be any three linguistic distribution assessments of a linguistic term set $S$.

Then the distance measure defined by Eq. (7) satisfies the following properties:

1. $d\left(m_{1}, m_{2}\right) \geq 0$;

2. $d\left(m_{1}, m_{2}\right)=d\left(m_{2}, m_{1}\right)$;

3. $d\left(m_{1}, m_{2}\right)=0$ if and only if $m_{1}=m_{2}$, i.e., $\beta_{k}^{1}=\beta_{k}^{2}(k=0,1, \ldots, g-1)$;

$d\left(m_{1}, m_{2}\right)=1$ if and only if $m_{1}=m_{\min }\left(\operatorname{orm}_{\max }\right)$, $m_{2}=m_{\text {max }}\left(\right.$ or $\left.m_{\text {min }}\right)$

where $m_{\min }=\left\{\left(s_{0}, 1\right), \ldots,\left(s_{g-1}, 0\right)\right\}$ and $m_{\max }=$ $\left\{\left(s_{0}, 0\right), \ldots,\left(s_{g-1}, 1\right)\right\}$;

4. $\quad d\left(m_{1}, m_{2}\right) \leq d\left(m_{1}, m_{3}\right)+d\left(m_{3}, m_{2}\right)$.

Proof. (1) and (2) are obvious.

(3). According to Definition 3.4, $d\left(m_{1}, m_{2}\right)=0$ holds if and only if $\frac{1}{g-1} \sum_{k=0}^{g-1}\left|\sum_{r=0}^{k} \beta-\sum_{r=0}^{k} \beta_{r}^{2}\right|=0$, which means that $\left|\sum_{r=0}^{k} \beta_{r}^{1}-\sum_{r=0}^{k} \beta_{r}^{2}\right|=$ $0, k=0,1, \ldots, g-1$. For $k=0$, we can obtain $\beta_{0}^{1}=\beta_{0}^{2}$; For $k=0,1$, we can obtain $\beta_{1}^{1}=\beta_{1}^{2} ; \cdots$; For $k=0,1, \ldots, g-1$, we can obtain $\beta_{g-1}^{1}=\beta_{g-1}^{2}$. Therefore, $d\left(m_{1}, m_{2}\right)=0$ if and only if $m_{1}=m_{2}$. For the second part, on the one hand, if $m_{1}=\left\{\left(s_{0}, 1\right), \ldots,\left(s_{g-1}, 0\right)\right\}$, $m_{2}=\left\{\left(s_{0}, 0\right), \ldots,\left(s_{g-1}, 1\right)\right\}$ or $m_{1}=\left\{\left(s_{0}, 0\right), \ldots,\left(s_{g-1}, 1\right)\right\}, m_{2}=$ $\left\{\left(s_{0}, 1\right), \ldots,\left(s_{g-1}, 0\right)\right\}$, one can get $d\left(m_{1}, m_{2}\right)=1$ by using the distance measure in Definition 3.4. On the other hand, if $d\left(m_{1}, m_{2}\right)=$ 1, we know that $\frac{1}{g-1} \sum_{k=0}^{g-1}\left|\sum_{r=0}^{k} \beta_{r}^{1}-\sum_{r=0}^{k} \beta_{r}^{2}\right|=1$. Since $0 \leq \mid \sum_{r=0}^{k} \beta_{r}^{1}-$ $\sum_{r=0}^{k} \beta_{r}^{2} \mid \leq 1$, we can obtain $\left|\sum_{r=0}^{k} \beta_{r}^{1}-\sum_{r=0}^{k} \beta_{r}^{2}\right|=1, k=0,1, \ldots, g-1$. Notice that $0 \leq \sum_{r=0}^{k} \beta_{r}^{1} \leq 1,0 \leq \sum_{r=0}^{k} \beta_{r}^{2} \leq 1, k=0,1, \ldots, g-1$. Hence, we have $\sum_{r=0}^{k} \beta_{r}^{1}=1$ and $\sum_{r=0}^{k} \beta_{r}^{1}=0, k=0,1, \ldots, g-1$ or $\sum_{r=0}^{k} \beta_{r}^{1}=0$ and $\sum_{r=0}^{k} \beta_{r}^{1}=1, k=0,1, \ldots, g-1$. Since $\sum_{r=0}^{k} \beta_{r}^{1} \geq$

$\sum_{r=0}^{k-1} \beta_{r}^{1}, \sum_{r=0}^{k} \beta_{r}^{2} \geq \sum_{r=0}^{k-1} \beta_{r}^{2}, k=1, \ldots, g-1$, we can conclude that

Table $1 \mid$ Calculation results of different distance measure for linguistic distribution assessments.

\begin{tabular}{lllll}
\hline & $\begin{array}{l}\text { Equation (4) } \\
\text { Zhang } \text { et al. [7] }\end{array}$ & $\begin{array}{l}\text { Equation (5) } \\
\text { Zhang } \text { et al. [14] }\end{array}$ & $\begin{array}{l}\text { Equation (6) } \\
\text { Yu } \text { et al. [15] }\end{array}$ & $\begin{array}{l}\text { Equation (7) The } \\
\text { Proposed Measure }\end{array}$ \\
\hline Example 1 & $d\left(m_{1}, m_{2}\right)=1$, & $d\left(m_{1}, m_{2}\right)=0.50$, & $d\left(m_{1}, m_{2}\right)=0.21$, & $d\left(m_{1}, m_{2}\right)=0.50$, \\
& $d\left(m_{1}, m_{3}\right)=1$ & $d\left(m_{1}, m_{3}\right)=0.83$ & $d\left(m_{1}, m_{3}\right)=0.38$ & $d\left(m_{1}, m_{3}\right)=0.83$ \\
Example 2 & $d\left(m_{1}, m_{2}\right)=0.4$ & $d\left(m_{1}, m_{2}\right)=0$ & $d\left(m_{1}, m_{2}\right)=0.06$ & $d\left(m_{1}, m_{2}\right)=0.10$ \\
Example 3 & $d\left(m_{1}, m_{2}\right)=1$, & $d\left(m_{1}, m_{2}\right)=0.55$, & $d\left(m_{1}, m_{2}\right)=0.16$, & $d\left(m_{1}, m_{2}\right)=0.55$, \\
& $d\left(m_{1}, m_{3}\right)=1$ & $d\left(m_{1}, m_{3}\right)=0.38$ & $d\left(m_{1}, m_{3}\right)=0.20$ & $d\left(m_{1}, m_{3}\right)=0.38$ \\
\hline
\end{tabular}


$\beta_{0}^{1}=\beta_{g-1}^{2}=1, \beta_{1}^{1}=\ldots=\beta_{g-1}^{1}=\beta_{0}^{2}=\ldots=\beta_{g-2}^{2}=0$ or $\beta_{g-1}^{1}=\beta_{0}^{2}=1, \beta_{0}^{1}=\ldots=\beta_{g-2}^{1}=\beta_{1}^{2}=\ldots=\beta_{g-1}^{2}=0$, i.e., $m_{1}=m_{\min }\left(\right.$ or $\left.m_{\max }\right), m_{2}=m_{\max }\left(\right.$ or $\left._{\min }\right)$.

(4) Notice that $d\left(m_{1}, m_{2}\right)=\frac{1}{g-1} \sum_{k=0}^{g-1} \mid \sum_{r=0}^{k} \beta_{r}^{1}-$ $\sum_{r=0}^{k} \beta_{r}^{2}\left|=\frac{1}{g-1} \sum_{k=0}^{g-1}\right| \sum_{r=0}^{k} \beta_{r}^{1}-\sum_{r=0}^{k} \beta_{r}^{3}+\sum_{r=0}^{k} \beta_{r}^{3}-\sum_{r=0}^{k} \beta_{r}^{2} \mid$ $\leq \quad \frac{1}{g-1} \sum_{k=0}^{g-1}\left(\left|\sum_{r=0}^{k} \beta_{r}^{1}-\sum_{r=0}^{k} \beta_{r}^{3}\right|+\left|\sum_{r=0}^{k} \beta_{r}^{3}-\sum_{r=0}^{k} \beta_{r}^{2}\right|\right)=$ $\frac{1}{g-1} \sum_{k=0}^{g-1}\left|\sum_{r=0}^{k} \beta_{r}^{1}-\sum_{r=0}^{k} \beta_{r}^{3}\right|+\frac{1}{g-1} \sum_{k=0}^{g-1}\left|\sum_{r=0}^{k} \beta_{r}^{3}-\sum_{r=0}^{k} \beta_{r}^{2}\right|=$ $d\left(m_{1}, m_{3}\right)+d\left(m_{3}, m_{2}\right)$, i.e., $d\left(m_{1}, m_{2}\right) \leq d\left(m_{1}, m_{3}\right)+d\left(m_{3}, m_{2}\right)$.

This completes the proof of Theorem 3.1.

Definition 3.5. Let $m_{1}=\left\{\left(s_{k}, \beta_{k}^{1}\right) \mid k=0,1, \ldots, g-1\right\}$ and $m_{2}=$ $\left\{\left(s_{k}, \beta_{k}^{2}\right) \mid k=0,1, \ldots, g-1\right\}$ be two linguistic distribution assessments of a linguistic term set $S$, then the similarity degree between two linguistic distribution assessments $m_{1}$ and $m_{2}$ can be defined as

$$
\begin{aligned}
s\left(m_{1}, m_{2}\right) & =1-d\left(m_{1}, m_{2}\right) \\
& =1-\frac{1}{g-1} \sum_{k=0}^{g-1}\left|\sum_{r=0}^{k} \beta_{r}^{1}-\sum_{r=0}^{k} \beta_{r}^{2}\right| .
\end{aligned}
$$

Obviously, $0 \leq s\left(m_{1}, m_{2}\right) \leq 1$. The closer $s\left(m_{1}, m_{2}\right)$ is to 1 , the more similar $m_{1}$ is to $m_{2}$, while the closer $s\left(m_{1}, m_{2}\right)$ is to 0 , the more distant $m_{1}$ is from $m_{2}$.

\section{A CONSENSUS SUPPORT MODEL FOR MAGDM WITH LINGUISTIC DISTRIBUTION ASSESSMENTS}

In this subsection, we describe the framework of the proposed consensus reaching model for MAGDM with linguistic distribution assessments.

\subsection{The Framework of the Proposed Consensus Support Model}

Consider a MAGDM problem with linguistic distribution assessments. Let $X=\left\{x_{1}, x_{2}, \cdots, x_{m}\right\}$ be a discrete set of $m(m \geq 2)$ potential alternatives, $C=\left\{c_{1}, c_{2}, \cdots, c_{n}\right\}$ be the set of $n(n \geq 2)$ attributes or criteria. Suppose that $W=\left\{w_{1}, w_{2}, \cdots, w_{n}\right\}^{T}$ is the weight vector of attributes, such that $\sum_{j=1}^{n} w_{j}=1, w_{j} \geq 0, j \in\{1,2, \ldots, n\}$ and $w_{j}$ denotes the weight of attribute $c_{j}$. There are a group of experts, $E=\left\{e_{1}, e_{2}, \cdots, e_{T}\right\}$. Assume $\lambda=\left\{\lambda_{1}, \lambda_{2}, \cdots, \lambda_{T}^{T}\right\}$ is the weight vector of the experts, where $0 \leq \lambda_{t} \leq 1, t \in\{1,2, \ldots, T\}$ and $\sum_{t=1}^{T} \lambda_{t}=1$. Suppose that $M_{t}=\left(m_{i j, t}\right)_{m \times n}$ is the linguistic distribution assessment decision matrix given by the expert $e_{t} \in E$, where $m_{i j, t}=\left\{\left\langle s_{k}, p_{i j, t}^{k}\right\rangle \mid k=0,1, \cdots, g-1\right\}$ represents the performance of the alternative $x_{i}$ with respect to the attribute $c_{j}$. The aim of MAGDM is to select or prioritize these finite alternatives with a consensus.

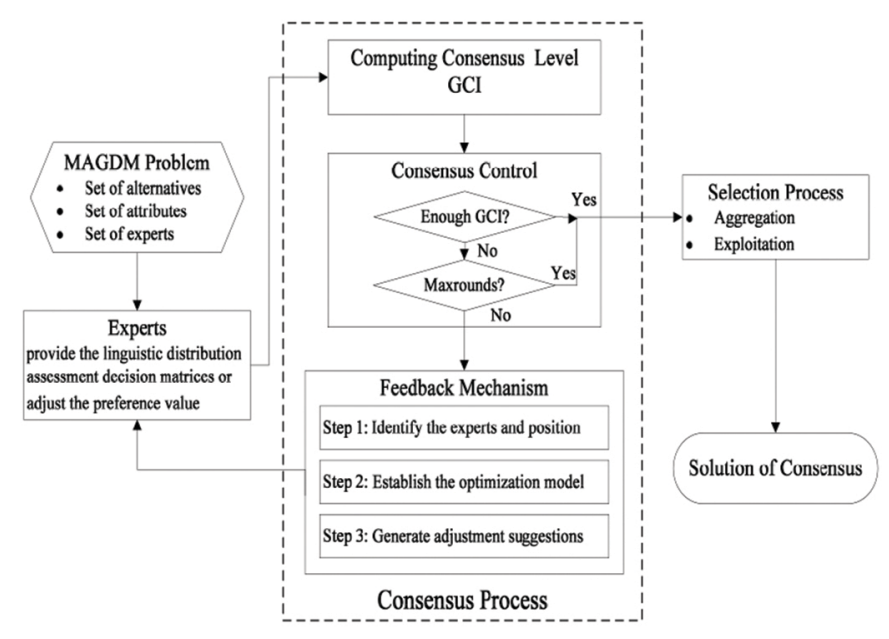

Figure 2 The resolution framework for multi-attribute group decisionmaking (MAGDM) problem with LDA.

Remark 1. In the considered MAGDM problem, it is assumed that preferences of experts are represented by using linguistic distribution assessments. Although linguistic distribution assessment can be used to enhance the flexibility and richness of linguistic elicitation by experts in hesitant situations under qualitative settings, experts may have some cognitive difficulties in using linguistic distribution assessments to articulate their preferences in practice. Especially, experts may find it difficult to provide proportional distribution information on HFLTS with too many linguistic terms. Therefore, in practice, it is suggested that experts should provide the proportional distribution information on HFLTS with no more than three linguistic terms when articulating preferences by using linguistic distribution assessments.

A typical resolution method for a GDM problem consists of two different processes: the consensus process and the selection process. The consensus process refers to how to obtain the maximum degree of consensus or agreement among the experts on the solution alternatives, while the selection process consists in how to obtain the solution set of alternatives from the opinions on the alternatives given by the experts. Inspired by these two processes, we propose the framework of the MAGDM with linguistic distribution assessments. The details of the framework are described in Figure 2. As we know, the feedback mechanism is the core part of a consensus reaching model. There are three main steps in the proposed feedback mechanism. In the first step, an IR is implemented to locate the experts who need to reevaluate his/her assessment values and the position where he/she need to modify. In the second step, an optimization model is established to minimize the deviation between the adjusted values and initial preferences. Simultaneously, additional preference information provided by the experts is also incorporated into the optimization model. By solving the constructed optimization model, adjustment advices are generated in the third step. The details of the consensus process are introduced in the following subsections.

\subsection{The Consensus Measure}

The weighted averaging operator $D A W A_{\lambda}$ can be used to transform individual linguistic distribution assessment decision matrix 
$M_{t}=\left(m_{i j, t}\right)_{m \times n}, t \in\{1,2, \ldots, T\}$ into the following collective decision matrix $M_{c o l}=\left(m_{i j, c o l}\right)_{m \times n}$ :

$$
M_{c o l}=\left(\begin{array}{llll}
m_{11, c o l} & m_{12, c o l} & \cdots & m_{1 n, c o l} \\
m_{21, c o l} & m_{22, c o l} & \cdots & m_{2 n, c o l} \\
\cdots & & & \\
m_{m 1, c o l} & m_{m 2, c o l} & \cdots & m_{m n, c o l}
\end{array}\right) \text {, }
$$

where

$$
\begin{aligned}
m_{i j, c o l} & =\left\{\left\langle s_{k}, p_{i j, c o l}^{k}\right\rangle \mid k=0,1, \cdots, g-1\right\} \\
& =D A W A_{\lambda}\left(m_{i j, 1}, m_{i j, 2}, \ldots, m_{i j, T}\right) \\
& =\left\{\left\langle s_{k}, \sum_{t=1}^{T} \lambda_{t} p_{i j, t}^{k}\right\rangle \mid k=0,1, \cdots, g-1\right\}
\end{aligned}
$$

Based on the similarity degree of two linguistic distribution assessments, for each expert $e_{t}(t=1,2, \ldots, T)$, a similarity matrix $S^{t, c o l}=$ $\left(s_{i j}^{t, c o l}\right)_{m \times n}$ can be defined by

$$
S^{t, c o l}=\left(\begin{array}{lllc}
s_{11}^{t, c o l} & s_{12}^{t, c o l} & \cdots & s_{1 n}^{t, c o l} \\
s_{21}^{t, c o l} & s_{22}^{t, c o l} & \ldots & s_{2 n}^{t, c o l} \\
\cdots & & & \\
s_{m 1}^{t, c o l} & s_{m 2}^{t, c o l} & \cdots & s_{m n}^{t, c o l}
\end{array}\right),
$$

where $s_{i j}^{t, c o l}$ is the similarity between the expert $e_{t}$ and the group in their assessments of the position $(i, j), i=1,2, \ldots, m ; j=$ $1,2, \ldots, n$. That is,

$$
\begin{aligned}
s_{i j}^{t, c o l} & =1-d\left(m_{i j, t}, m_{i j, c o l}\right) \\
& =1-\frac{1}{g-1} \sum_{k=0}^{g-1}\left|\sum_{r=0}^{k} p_{i j, t}^{r}-\sum_{r=0}^{k} p_{i j, c o l}^{r}\right| .
\end{aligned}
$$

There are $T$ similarity matrices. By aggregating all the similarity matrices $S^{t, c o l}(t=1,2, \ldots, T)$ associated with each expert $e_{t}$, we can obtain a consensus matrix $S^{c o l}=\left(s_{i j}^{c o l}\right)_{m \times n}$ as follows:

$$
S^{c o l}=\left(\begin{array}{cccc}
s_{11}^{c o l} & s_{12}^{c o l} & \cdots & s_{1 n}^{c o l} \\
s_{21}^{c o l} & s_{22}^{c o l} & \cdots & s_{2 n}^{c o l} \\
\cdots & & & \\
s_{m 1}^{c o l} & s_{m 2}^{c o l} & \cdots & s_{m n}^{c o l}
\end{array}\right) .
$$

The aggregation is conducted using an weighted aggregation operator Agg,

$$
s_{i j}^{c o l}=\operatorname{Agg}\left(s_{i j}^{1, c o l}, \ldots, s_{i j}^{T, c o l}\right)=\sum_{t=1}^{T} \lambda_{t} s_{i j}^{t, c o l} .
$$

In the following, based on the consensus matrix, the group consensus index can be defined.
Definition 4.1. The group consensus index GCI for the decision matrices $M_{t}(t=1,2, \ldots, T)$ is defined as

$$
G C I=\min \left\{s_{i j}^{c o l} \mid 1 \leq i \leq m, 1 \leq j \leq n\right\} .
$$

The group consensus index GCI is defined to measure the overall consensus level among the decision makers' decision matrices and is used to control the process of the consensus reaching. Although the group consensus level GCI can be calculated using the arithmetic mean of all the consensus degrees on the attribute values, here we adopt a min operator to allow for a more rigorous criterion. In this way, a compromise is avoided between some attribute values with very high consensus degrees and those with very low consensus degrees.

Obviously, $0 \leq G C I \leq 1$. The larger the value of $G C I$, the closer that the experts are to each others. The threshold of consensus level $\overline{G C I}$ can be determined by the experts in advance according to the actual situation. When $G C I \geq \overline{G C I}$, it can be concluded that an acceptable level of consensus is achieved among the experts.

\subsection{The Feedback Mechanism}

When comparing the current consensus level GCI with the predefined consensus threshold value $\overline{G C I}$, if $G C I \geq \overline{G C I}$, then the CRP ends; otherwise, a new interaction should be conducted. The feedback mechanism generates personalized suggestions to the decision makers according to the consensus criteria. It helps decision makers to modify their preference values with respect to attributes. The proposed feedback mechanism consists of two advice rules: IRs and recommendation rule (RR).

(a) Identification rules: The IRs are utilized to identify attribute values provided by the experts that are contributing less to the attainment of a high consensus level. In the CRP for MAGDM, the IR are given to locate which expert need to reevaluate his/her assessment values and in which specific position he/she need to modify. Here, we define the set IDE that contains 3-tuples $(t, i, j)$ symbolizing attribute values $m_{i j, t}$ that should be changed because they affect badly to that consensus state. To compute IDE, we apply a two step identification process that uses the consensus measures previously defined.

\section{Identification of position $(i, j)$ :}

$$
P O S=\left\{(i, j) \mid s_{i j}^{c o l}=\min \left\{s_{i j}^{c o l} \mid 1 \leq i \leq m, 1 \leq j \leq n\right\}\right\}
$$

2. Identification of expert-position $(t, i, j)$ :

$$
I D E=\left\{(t, i, j) \mid s_{i j}^{t, c o l}<\overline{G C I} \wedge(i, j) \in P O S\right\}
$$

(b) Recommendation rules: The RRs are to generate personalized advices to help experts to change their evaluation matrices. For each $(t, i, j) \in I D E$, the RRs will give recommendations for adjusting the corresponding attribute value. The RRs of the proposed model is based on an optimization model. At each iteration of our method, an optimization model is established for each $(i, j) \in$ POS. By solving the optimization model, suggestions will be generated for all experts who need to adjust preferences in the position $(i, j)$. The model proposed in this paper has two prominent 
characteristics. Firstly, the adjustment of attribute values takes into account the additional preference information from the experts. Secondly, under the condition that the group consensus level is improved, the optimization model protects the expert initial preference information by minimizing the adjustments.

Let $E_{i j}^{M}=\left\{t \mid s_{i j}^{t, c o l}<\overline{G C I}\right\}$ be the set of subscripts of experts who need to modify the values in the position $(i, j) \in P O S$. Let $\sharp E_{i j}^{M}$ denote the number of elements contained in the set $\sharp E_{i j}^{M}$. At each iteration, there may be one or more experts who need to modify their preferences, that is, $\sharp E_{i j}^{M} \geq 1$ for $(i, j) \in P O S$. Since the attribute values is in the form of linguistic distribution assessments, the modification of attribute value $m_{i j, t}=\left\{\left\langle s_{k}, p_{i j, t}^{k}\right\rangle \mid k=0,1, \cdots, g-1\right\}$ is essentially to adjust the distribution assessment $p_{i j, t}=\left(p_{i j, t}^{0}, p_{i j, t}^{1}, \cdots, p_{i j, t}^{g-1}\right)$. Let $d_{i j, t}^{-}=$ $\left(d_{i j, t}^{0,-}, d_{i j, t}^{1,-}, \cdots, d_{i j, t}^{g-1,-}\right)$ and $d_{i j, t}^{+}=\left(d_{i j, t}^{0,+}, d_{i j, t}^{1,+}, \cdots, d_{i j, t}^{g-1,+}\right)$ be the vector of negative deviations and positive deviations, respectively. The proposed RRs are based on the following optimization model $(M-1)$ :

$$
\begin{aligned}
& (M-1) \\
& \operatorname{Min} D=\sum_{t \in T_{i j}^{M}} \operatorname{dev}\left(m^{\prime}{ }_{i j, t}, m_{i j, t}\right) \\
& \text { s.t. }\left\{\begin{array}{l}
G C I^{\prime}>G C I \\
d_{i j, t}^{-} \in F_{i j, t}^{-}, t \in E_{i j}^{M}, \\
d_{i j, t}^{+} \in F_{i j, t}^{+}, t \in E_{i j}^{M}
\end{array},\right.
\end{aligned}
$$

where $\operatorname{dev}\left(m_{i j, t}^{\prime}, m_{i j, t}\right)$ is the deviation between the adjusted linguistic distribution assessment $m_{i j, t}^{\prime}$ and the original linguistic distribution assessment $m_{i j, t}$, GCI and GCI are the group consensus indices after and beforeadjustment, respectively, and $F_{i j, t}^{-}$and $F_{i j, t}^{+}$ are the feasible sets of $d_{i j, t}^{-}$and $d_{i j, t}^{+}$. In model $(M-1)$, the objective is to minimize the sum of deviations, so that the original preference information of the experts can be retained to the largest possible extent. The purpose of imposing constraint condition, $G C I^{\prime}>$ $G C I$, on model $(M-1)$ is to improve the group consensus level. Notice that $(i, j) \in P O S$ means $G C I=s_{i j}^{c o l}$, which means that the increase of $s_{i j}^{c o l}$ will lead to the improvement of group consensus level. In order to handle the constraint of improvement effectively, we transform the model $(M-1)$ into the following model $(M-2)$ by increasing the group similarity in the position $(i, j)$ :

$$
\begin{aligned}
& (M-2) \\
& \operatorname{Min} D=\sum_{t \in T_{i j}^{M}} \operatorname{dev}\left(m_{i j, t}^{\prime}, m_{i j, t}\right) \\
& \text { s.t. }\left\{\begin{array}{l}
\sum_{t=1}^{T} \lambda_{t} s\left(m_{i j, t}^{\prime}, m_{i j, c o l}^{\prime}\right) \geq \Delta_{i j} \\
d_{i j, t}^{-} \in F_{i j, t}^{-}, t \in T_{i j}^{M} \\
d_{i j, t}^{+} \in F_{i j, t}^{+}, t \in T_{i j}^{M}
\end{array},\right.
\end{aligned}
$$

where $\triangle_{i j}$ is a proper parameter for controlling the group similarity level in the position $(i, j)$ after adjustment. To ensure an adequate improvement of the group consensus level, we set the value of the parameter $\triangle_{i j}$ as

$$
\Delta_{i j}=\min \left\{\overline{G C I}, \min _{(2)}\left\{s_{i j}^{c o l}\right\}+\varepsilon\right\},
$$

where $\min _{(2)}\left\{s_{i j}^{c o l}\right\}$ is the second smallest value in the consensus matrix $S^{c o l}$, and $\varepsilon$ is a small positive number.

$\Delta_{i j}$ determined by the Equation (17) can guarantee that after the adjustment of preference values, the group consensus level in the position $(i, j)$ can either reach the threshold $\overline{G C I}$ or exceed the second smallest value in the consensus matrix $S^{c o l}$. In other words, it ensures that the group consensus level can be adequately improved by using $\Delta_{i j}$ determined by the Equation (17) at each iteration. We also note that the positive number $\varepsilon$ is an important parameter in CRP. Under the premise that the model has solutions, a larger $\varepsilon$ will reduce the number of iterations to reach consensus. But on the other hand, when $\varepsilon$ is too large, there may be no solution to the optimization problem. Therefore, there is a trade-off between iterative speed and solvability in determining the value of $\varepsilon$.

In order to reflect the real preferences of decision makers, the proposed consensus reaching model allows decision makers to provide additional preference information at each iteration. It is supposed in our model that decision makers are willing to provide preference information about deviations $d_{i j, t}^{k,-}$ or/and $d_{i j, t}^{k,+}$. For $(i, j) \in P O S$, denote

$$
B_{i j, t}^{-}=\left(B_{i j, t}^{0,-}, \ldots, B_{i j, t}^{g-1,-}\right), t \in E_{i j}^{M}
$$

and

$$
B_{i j, t}^{+}=\left(B_{i j, t}^{0,+}, \ldots, B_{i j, t}^{g-1,+}\right), t \in E_{i j}^{M},
$$

where $B_{i j, t}^{k,-}=\max \left\{0, p_{i j, t}^{k}-p_{i j, c o l}^{k}\right\}(k=0,1, \ldots, g-1)$ and $B_{i j, t}^{k,+}=$ $\max \left\{0, p_{i j, c o l}^{k}-p_{i j, t}^{k}\right\}\left(k=0,1, \ldots, g-1 ; t \in E_{i j}^{M}\right)$.

Clearly, the variables $d_{i j, t}^{k,-}$ and $d_{i j, t}^{k,+}$ have to meet $0 \leq d_{i j, t}^{k,-} \leq B_{i j, t}^{k,-}$ and $0 \leq d_{i j, t}^{k,+} \leq B_{i j, t}^{k,+}$ in order to improve the consensus level. Therefore, the vectors $B_{i j, t}^{-}$and $B_{i j, t}^{+}$can be used to elicit additional preference information from the decision maker $e_{t}$. In particular, $B_{i j, t}^{-}$and $B_{i j, t}^{+}$ can be regarded as the upper bounds for determining the ranges of $d_{i j, t_{0}}^{k, L B}$ and $d_{i j, t_{0}}^{k, U B}$. Suppose that the decision maker $e_{t}$ provides the maximum adjustment $d_{i j, t_{0}}^{k, L B} \in\left[0, B_{i_{0} j_{0}, t}^{k,-}\right]$ for the decision variable $d_{i j, t_{0}}^{k,-}$, as well as the maximum adjustment $d_{i j, t_{0}}^{k, U B} \in\left[0, B_{i_{0} j_{0}, t}^{k,+}\right]$ for the decision variable $d_{i j, t_{0}}^{k,+}$. By specifying the constraints on the variables $d_{i j, t}^{k,-}$ and $d_{i j, t}^{k,+}$, we can obtain the following model $(M-3)$ :

$$
\begin{aligned}
& \text { Min } Z=\sum_{t \in T_{i j}^{M}} \lambda_{t} \sum_{k=0}^{g-1}\left(d_{i j, t}^{k,-}+d_{i j, t}^{k,+}\right) \\
& \text { s.t. }\left\{\begin{array}{l}
\sum_{t=1}^{T} \lambda_{t} d\left(m^{\prime}{ }_{i j, t}, m^{\prime}{ }_{i j, c o l}\right) \leq 1-\Delta_{i j} \\
\sum_{k=0}^{g-1} d_{i j, t}^{k,+}-\sum_{k=0}^{g-1} d_{i j, t}^{k,-}=0, t \in T_{i j}^{M} \\
0 \leq d_{i j, t}^{k,-} \leq \min \left\{d_{i j, t}^{k, L B}, p_{i j, t}^{k}\right\} \\
0 \leq d_{i j, t}^{k,+} \leq \min \left\{d_{i j, t}^{k, U B}, 1-p_{i j, t}^{k}\right\}
\end{array}\right.
\end{aligned}
$$


In model $(M-3)$, the objective is to minimize the weighted sum of all deviation variables for $t \in T_{i j}^{M}$. The second constraint guarantees that the adjusted distribution still satisfies the normalization condition, that is, $\sum_{k=0}^{g-1}\left(p_{i j, t}^{k}-d_{i j, t}^{k,-}+d_{i j, t}^{k,+}\right)=1$. The third and the fourth constraints are the restrictions on the additional preference information provided by decision makers, as well as the nonnegativity of the adjusted distribution.

\subsection{An Iterative Algorithm for CRP}

For a MAGDM problem, let $M_{t}=\left(m_{i j, t}\right)_{m \times n}$ be the decision matrix given by the expert $e_{t} \in E$, where $m_{i j, t}=$ $\left\{\left\langle s_{k}, p_{i j, t}^{k}\right\rangle \mid k=0,1, \cdots, g-1\right\}$ is linguistic distribution assessment representing the performance of the alternative $x_{i}$ with respect to the attribute $c_{j}$. Suppose that $W=\left\{w_{1}, w_{2}, \cdots, w_{n}\right\}^{T}$ is the weight vector of attributes, such that $\sum_{j=1}^{n} w_{j}=1, w_{j} \geq 0, j \in\{1,2, \ldots, n\}$ and $\lambda=\left\{\lambda_{1}, \lambda_{2}, \text { cdots }, \lambda_{T}\right\}^{T}$ is the weight vector of the experts, where $0 \leq \lambda_{t} \leq 1, t \in\{1,2, \ldots, T\}$ and $\sum_{t=1}^{T} \lambda_{t}=1$. Assume Round $_{\max }$ is the maximum number of iterative times, $\overline{G C I}$ is the threshold of group consensus level. Let $\varepsilon$ be a small positive number. Let $l$ be the number of the iterative times. To reach a predefined consensus level $\overline{G C I}$, we propose the following algorithm.

Algorithm 1. (Consensus reaching process)

Input: $M_{t}=\left(m_{i j, t}\right)_{m \times n}, e_{t} \in E$, the maximum number of iterative times Round $_{\text {max }}$, the threshold of group consensus level $\overline{G C I}$.

Output: Improved decision matrices $\overline{M_{t}}=\left(\overline{m_{i j, t}}\right)_{m \times n}, e_{t} \in E$ and the iterative times $\bar{l}$.

Step 1. Initiate the procedure. Let $l=0$ and $M_{t}^{(0)}=\left(m_{i j, t}^{(0)}\right)_{m \times n}=$ $M_{t}=\left(m_{i j, t}\right)_{m \times n}, t=1,2, \ldots, T$.

Step 2. Utilize the operator $D A W A_{\lambda}$ to fuse all individual decision matrices $M_{t}^{(l)}=\left(m_{i j, t}^{(l)}\right)_{m \times n}(t=1,2, \ldots, T)$ into a collective decision matrix $M_{c o l}^{(l)}=\left(m_{i j, c o l}^{(l)}\right)_{m \times n}$, where $m_{i j, c o l}^{(l)}=$ $D A W A_{\lambda}\left(m_{i j, 1}^{(l)}, m_{i j, 2}^{(l)}, \ldots, m_{i j, T}^{(l)}\right)$.

Step 3. Calculate the similarity matrix $S_{(l)}^{t, c o l}=\left(s_{i j,(l)}^{t, c o l}\right)_{m \times n}$ between each individual decision matrix $M_{t}^{(l)}=\left(m_{i j, t}^{(l)}\right)_{m \times n}$ and the collective decision matrix $M_{c o l}^{(l)}=\left(m_{i j, c o l}^{(l)}\right)_{m \times n}$ by using (12).

Step 4. Aggregate all the similarity matrices $S_{(l)}^{t, c o l}(t=1,2, \ldots, T)$ into the consensus matrix $S_{(l)}^{c o l}=\left(s_{i j,(l)}^{c o l}\right)_{m \times n}$ by using Equation (14).

Step 5. Determine the group consensus index $G C I^{(l)}$ by using (15). if $G C I^{(l)} \geq \overline{G C I}$ or $l \geq$ Round $_{\text {max }}$, then go to Step 11, otherwise, go to the next step.
Step 6. Identify attribute values $m_{i j, t}^{(l)}$ that should be adjusted by using theIRs.

Locate the position $(i, j)$ by using (16), that is,

$$
\operatorname{POS}_{l}=\left\{(i, j) \mid s_{i j,(l)}^{c o l}=\min \left\{s_{i j,(l)}^{c o l} \mid 1 \leq i \leq m, 1 \leq j \leq n\right\}\right\},
$$

and determine the set

$$
E_{i j,(l)}^{M}=\left\{t \mid s_{i j,(l)}^{t, c o l}<\overline{G C I}\right\} .
$$

Let $I D E_{(l)}$ be the set that contains 3-tuples $(t, i, j)$ symbolizing attribute values $m_{i j, t}^{(l)}$ that should be changed.

Step 7. Elicit preference information about $d_{i j, t}^{k,-}$ and $d_{i j, t}^{k,+}$ from the decision maker $e_{t}\left(t \in E_{i j,(l)}^{M}\right)$. In this step, the vectors $B_{i j, t}^{-,(l)}$ and $B_{i j, t}^{+,(l)}$ is calculated by using Eqs. (19) and (20). Based on $B_{i j, t}^{-,(l)}$ and $B_{i j, t}^{+,(l)}$, the decision maker $e_{t}$ provides $d_{i j, t}^{k, L B} \in\left[0, B_{i j, t}^{k,-}\right]$ and $d_{i j, t}^{k, U B} \ln \left[0, B_{i j, t}^{k,+}\right]$ as the ranges of the variables $d_{i j, t}^{k,-}$ and $d_{i j, t}^{k,+}$.

Step 8. Solve the optimization model $(M-3)$. Denote the optimal solution of model $(M-3)$ as $d_{i j, t}^{k,-*}$ and $d_{i j, t}^{k,+*}, k=0,1, \ldots, g-1$.

Step 9. Adjust the linguistic distribution assessments $m_{i j, t}^{(l)},(i, j) \in$ $\left.\operatorname{POS}_{(l)}, t \in E_{i j,(l)}^{M}\right)$. Let $m_{i j, t}^{(l) \prime}=\left\{\left\langle s_{k}, p_{i j, t}^{k,(l) \prime}\right\rangle \mid k=0,1, \cdots, g-1\right\}$, where $\left.p_{i j, t}^{k,(l) \prime}=p_{i j, t}^{k,(l)}-d_{i j, t}^{k,-*}+d_{i j, t}^{k,+*},(i, j) \in \operatorname{POS}_{(l)}, t \in E_{i j,(l)}^{M}\right), k=$ $0,1, \ldots, g-1$. And determine the updated decision matrices $M_{t}^{(l+1)}=$ $\left(m_{i j, t}^{(l+1)}\right)_{m \times n}(t=1,2, \ldots, T)$, where

$$
m_{i j, t}^{(l+1)}= \begin{cases}m_{i j, t}^{(l) \prime} & (i, j) \in P O S_{(l)}, t \in E_{i j,(l)}^{M} \\ m_{i j, t}^{(l)} & \text { otherwise }\end{cases}
$$

Step 10. Determine the updated consensus matrix $S_{(l+1)}^{c o l}=$ $\left(s_{i j,(l+1)}^{c o l}\right)_{m \times n}$. For $(i, j) \in P O S_{(l)}$, calculate $s_{i j,(l+1)}^{c o l}$ by repeating step $2-4$; otherwise, let $s_{i j,(l+1)}^{c o l}=s_{i j,(l)}^{c o l}$. Let $l=l+1$, and return to step 5 .

Step 11. Output $\overline{M_{t}}=\left(\overline{m_{i j, t}}\right)_{m \times n}=M_{t}^{(l)}=\left(m_{i j, t}^{(l)}\right)_{m \times n}, e_{t} \in E$ and $\bar{l}=l$.

\section{Step 12. End.}

The proposed Algorithm 1 is an iterative process which can improve the group consensus level. Regarding convergence of Algorithm 1, we can prove the following Theorem 4.1.

Theorem 4.1. Let $M_{t}=\left(m_{i j, t}\right)_{m \times n}$ be the $T$ individual decision matrices, let $S_{(l)}^{c o l}=\left(s_{i j,(l)}^{c o l}\right)_{m \times n}$ be the consensus matrices sequences generated in Algorithm 1, and let $G C I^{(l)}$ be the group consensus level sequences generated in Algorithm 1, then for each l, we have either $G C I^{(l+1)} \geq \overline{G C I}$ or $G C I^{(l+1)}>G C I^{(l)}$.

Proof. According to Definition 4.1 and Algorithm 1, we have

$$
G C I^{(l+1)}=\min \left\{s_{i j,(l+1)}^{c o l} \mid(i, j) \in \operatorname{POS}_{l}, \min _{(2)}\left\{s_{i j,(l)}^{c o l}\right\}\right\}
$$




$$
\begin{gathered}
\geq \min \left\{\Delta_{i j}^{(l)} \mid(i, j) \in \operatorname{POS}_{l}, \min _{(2)}\left\{s_{i j,(l)}^{c o l}\right\}\right\} \\
=\min \left\{\min \left\{\overline{G C I}, \min _{(2)}\left\{s_{i j,(l)}^{c o l}\right\}+\varepsilon\right\}, \min _{(2)}\left\{\begin{array}{c}
c o l \\
i j,(l)
\end{array}\right\}\right\} \\
=\min \left\{\overline{G C I}, \min _{(2)}\left\{s_{i j,(l)}^{c o l}\right\}\right\} .
\end{gathered}
$$

Therefore, if $\overline{G C I}<\min _{(2)}\left\{s_{i j,(l)}^{c o l}\right\}$, we have $G C I^{(l+1)} \geq \overline{G C I}$; otherwise, we have $G C I^{(l+1)} \geq \min _{(2)}\left\{s_{i j,(l)}^{c o l}\right\}>\min \left\{s_{i j,(l)}^{c o l}\right\}=G C I^{(l)}$, which completes the proof of the Theorem 4.1.

Theorem 4.1 shows that the group consensus level $G C I^{(l)}$ is increasing in the process of iteration. Since the parameter $\varepsilon$ is positive, we can always obtain an improved decision matrix for each expert, which satisfies the predefined consensus level $\overline{G C I}$ after finite times of implementing Algorithm 1.

Remark 2. From Algorithm 1, we can see that although additional preferences of experts are considered in the proposed CRP, experts don't need to provide preferences in the form of linguistic distribution assessments during iteration of CRP. In fact, experts only need to provide preferences about the adjustment range of the proportional distribution information on HFLTS.

\subsection{The Selection Process}

Once the group consensus level amongst experts has been achieved, we can obtain a group decision matrix $\overline{M_{c o l}}$ which represents the centralized opinions of the group. A selection process on the group decision matrix is applied to supply a selection set of alternatives.

Based on the updated collective decision matrix $\overline{M_{c o l}}=\left(\overline{m_{i j, c o l}}\right)_{m \times n}$, by using the known weights of attributes and $D A W A_{W}$ operator we can obtain the overall values of each alternative, that is, $\overline{m_{i, c o l}}=$ $D A W A_{W}\left\{\overline{m_{i 1, c o l}}, \overline{m_{i 2, c o l}}, \ldots, \overline{m_{\text {in,col }}}\right\}, i=1,2, \ldots, n$; then by using Equation (2), we can further obtain the expectation of each alternative, that is, $E\left(\overline{m_{i, c o l}}\right), i=1,2, \ldots, n$. At last, rank all the alternatives in descending order and select the best one(s) in accordance with the values of $E\left(\overline{m_{i, c o l}}\right), i=1,2, \ldots, n$.

\section{NUMERICAL ANALYSIS}

\subsection{Numerical Example}

In this section, an example of investment decision problem is used to illustrate the practicality and effectiveness of the proposed consensus-based decision- making model. In this example, an investment company wants to invest a sum of money in the best industrial sector. Suppose that there are four possible alternatives $A=\left\{A_{1}, A_{2}, A_{3}, A_{4}\right\}$, where $A_{1}$ is the car industry, $A_{2}$ is the food industry, $A_{3}$ is the computer industry, and $A_{4}$ is the weapons industry. To best serve the interests of shareholders, three experts $E=\left\{e_{1}, e_{2}, e_{3}\right\}$ from three departments within the company are invited to offer suggestions: $e_{1}$ is from the risk analysis department, $e_{2}$ from the growth analysis department, $e_{3}$ from the environmental impact analysis department.
Equal weights are assumed for the three experts. Each expert was asked to provide their assessments over the four candidates with respect to the following four attributes: $C_{1}$-the ability of sale, $C_{2}$-the ability of production, $C_{3}$-the ability of technology, $\mathrm{C}_{4}$-the ability of financing. The weight vector of attributes is $W=(0.25,0.25,0.25,0.25)$. Suppose that the experts evaluate the performance of each alternative by using linguistic distribution assessments. The linguistic term set used by the experts is $S=$ $\left\{s_{0}=\right.$ extremelypoor, $s_{1}=$ verypoor,$s_{2}=$ poor,$s_{3}=$ fair,$s_{4}=$ good , $s_{5}=$ verygood, $s_{6}=$ extremelygood $\}$. Individual decision matrices for each expert are listed in Tables 2-4. In this example, the following set of parameters is used: $\overline{G C I}=0.92, \varepsilon=0.005$.

Firstly, we use Algorithm 1 (CRP) to help experts achieve a consensus.

Step 1. Initiate the procedure. Let $l=0$, and $M_{1}^{(0)}=\left(m_{i j, 1}^{(0)}\right)_{m \times n}=$ $M_{1}, M_{2}^{(0)}=\left(m_{i j, 2}^{(0)}\right)_{m \times n}=M_{2}, M_{3}^{(0)}=\left(m_{i j, 3}^{(0)}\right)_{m \times n}=M_{3}$.

Step 2. Calculate the collective decision matrix. By using the operator $D A W A_{\lambda}$, the collective decision matrix is obtained and shown in Table 5.

Step 3. By using the similarity measure, we can obtain the similarity matrices between each individual decision matrix and the collective decision matrix. In the first round, we obtain the the similarity matrices $S_{(0)}^{1, c o l}, S_{(0)}^{2, c o l}, S_{(0)}^{3, c o l}$ as follows:

$$
\begin{aligned}
& S_{(0)}^{1, c o l}=\left(\begin{array}{llll}
0.9889 & 0.9889 & 0.9944 & 0.9444 \\
0.9556 & 0.9667 & 0.9333 & 0.9667 \\
0.9056 & 0.9500 & 0.9389 & 0.9556 \\
0.9556 & 0.9278 & 0.9500 & 0.9556
\end{array}\right) \\
& S_{(0)}^{2, c o l}=\left(\begin{array}{llll}
0.9667 & 0.9444 & 0.9778 & 0.8389 \\
0.9667 & 0.9389 & 0.8667 & 0.9833 \\
0.9222 & 0.9500 & 0.9944 & 0.9778 \\
0.9444 & 0.9556 & 0.9167 & 0.9778
\end{array}\right) \\
& S_{(0)}^{3, c o l}=\left(\begin{array}{llll}
0.9667 & 0.9556 & 0.9722 & 0.8944 \\
0.9444 & 0.9611 & 0.9333 & 0.9833 \\
0.8278 & 0.9667 & 0.9444 & 0.9778 \\
0.9778 & 0.9722 & 0.9667 & 0.9444
\end{array}\right)
\end{aligned}
$$

Step 4. By using Eq. (14), the similarity matrices can be aggregated into the consensus matrix $S_{(0)}^{\text {col }}$.

$$
S_{(0)}^{c o l}=\left(\begin{array}{cccc}
0.9741 & 0.9630 & 0.9815 & 0.8926 \\
0.9556 & 0.9556 & 0.9111 & 0.9778 \\
0.8852 & 0.9556 & 0.9593 & 0.9704 \\
0.9563 & 0.9519 & 0.9444 & 0.9593
\end{array}\right)
$$

Step 5. Based on the consensus matrix $S_{(0)}^{c o l}$, we can obtain the group consensus index $G C I=0.8852$ by using Equation (15). Since $G C I<\overline{G C I}$, some experts have to adjust their preferences.

Step 6. By using the IRs, we can locate the experts need to reevaluate his/her assessment values and specific position in which he/she need to modify. We obtain $I D E_{(0)}=\{(1,3,1),(3,3,1)\}$, which means that experts $e_{1}$ and $e_{3}$ should modify their preferences about alternative $A_{3}$ with respect to attribute $C_{1}$. 
Table 2 The individual linguistic distribution assessments matrix $M_{1}$.

\begin{tabular}{|c|c|c|c|c|}
\hline$m_{i j, 1}$ & $C_{1}$ & $C_{2}$ & $C_{3}$ & $C_{4}$ \\
\hline$A_{1}$ & $\begin{array}{l}m_{11,1}=\left\{\left(s_{2}, 0.2\right)\right. \\
\left.\left(s_{3}, 0.5\right),\left(s_{4}, 0.3\right)\right\}\end{array}$ & $\begin{array}{l}m_{12,1}=\left\{\left(s_{4}, 0.2\right)\right. \\
\left.\left(s_{5}, 0.6\right),\left(s_{6}, 0.2\right)\right\}\end{array}$ & $\begin{array}{l}m_{13,1}=\left\{\left(s_{3}, 0.3\right)\right. \\
\left.\left(s_{4}, 0.4\right),\left(s_{5}, 0.3\right)\right\}\end{array}$ & $\begin{array}{c}m_{14,1}=\left\{\left(s_{3}, 0.2\right)\right. \\
\left.\left(s_{4}, 0.6\right)\left(s_{5}, 0.2\right)\right\}\end{array}$ \\
\hline$A_{2}$ & $\begin{array}{l}m_{21,1}=\left\{\left(s_{3}, 0.6\right)\right. \\
\left.\left(s_{4}, 0.2\right),\left(s_{5}, 0.2\right)\right\}\end{array}$ & $\begin{array}{c}m_{22,1}=\left\{\left(s_{4}, 0.7\right)\right. \\
\left.\left(s_{5}, 0.3\right)\right\}\end{array}$ & $\begin{array}{l}m_{23,1}=\left\{\left(s_{2}, 0.4\right)\right. \\
\left.\left(s_{3}, 0.5\right),\left(s_{4}, 0.1\right)\right\}\end{array}$ & $\begin{array}{c}m_{24,1}=\left\{\left(s_{5}, 0.7\right)\right. \\
\left.\left(s_{6}, 0.3\right)\right\}\end{array}$ \\
\hline$A_{3}$ & $\begin{array}{c}m_{31,1}=\left\{\left(s_{4}, 0.3\right)\right. \\
\left.\left(s_{5}, 0.7\right)\right\}\end{array}$ & $\begin{array}{l}m_{32,1}=\left\{\left(s_{1}, 0.3\right)\right. \\
\left.\left(s_{2}, 0.6\right),\left(s_{3}, 0.1\right)\right\}\end{array}$ & $\begin{array}{l}m_{33,1}=\left\{\left(s_{2}, 0.5\right)\right. \\
\left.\left(s_{3}, 0.4\right),\left(s_{4}, 0.1\right)\right\}\end{array}$ & $\begin{array}{c}m_{34,1}=\left\{\left(s_{5}, 0.6\right)\right. \\
\left.\left(s_{6}, 0.4\right)\right\}\end{array}$ \\
\hline$A_{4}$ & $\begin{array}{l}m_{41,1}=\left\{\left(s_{2}, 0.2\right)\right. \\
\left.\left(s_{3}, 0.4\right),\left(s_{4}, 0.4\right)\right\}\end{array}$ & $\begin{array}{l}m_{42,1}=\left\{\left(s_{4}, 0.6\right)\right. \\
\left.\left(s_{5}, 0.2\right),\left(s_{6}, 0.2\right)\right\}\end{array}$ & $\begin{array}{l}m_{43,1}=\left\{\left(s_{3}, 0.4\right)\right. \\
\left.\left(s_{4}, 0.4\right),\left(s_{5}, 0.2\right)\right\}\end{array}$ & $\begin{array}{c}m_{44,1}=\left\{\left(s_{4}, 0.3\right)\right. \\
\left.\left(s_{5}, 0.7\right)\right\}\end{array}$ \\
\hline
\end{tabular}

Table 3 The individual linguistic distribution assessments matrix $M_{2}$.

\begin{tabular}{|c|c|c|c|c|}
\hline$A_{1}$ & $\begin{array}{l}m_{11,2}=\left\{\left(s_{2}, 0.3\right)\right. \\
\left.\left(s_{3}, 0.3\right),\left(s_{4}, 0.4\right)\right\}\end{array}$ & $\begin{array}{c}m_{12,2}=\left\{\left(s_{4}, 0.4\right)\right. \\
\left.\left(s_{5}, 0.6\right)\right\}\end{array}$ & $\begin{array}{l}m_{13,2}=\left\{\left(s_{3}, 0.3\right)\right. \\
\left.\left(s_{4}, 0.3\right),\left(s_{5}, 0.4\right)\right\}\end{array}$ & $\begin{array}{l}m_{14,2}=\left\{\left(s_{2}, 0.5\right),\right. \\
\left.\left(s_{3}, 0.3\right),\left(s_{4}, 0.2\right)\right\}\end{array}$ \\
\hline$A_{2}$ & $\begin{array}{l}m_{21,2}=\left\{\left(s_{2}, 0.1\right)\right. \\
\left.\left(s_{3}, 0.4\right),\left(s_{4}, 0.5\right)\right\}\end{array}$ & $\begin{array}{l}m_{22,2}=\left\{\left(s_{3}, 0.5\right)\right. \\
\left.\left(s_{4}, 0.2\right),\left(s_{5}, 0.3\right)\right\}\end{array}$ & $\begin{array}{l}m_{23,2}=\left\{\left(s_{3}, 0.3\right)\right. \\
\left.\left(s_{4}, 0.5\right),\left(s_{5}, 0.2\right)\right\}\end{array}$ & $\begin{array}{c}m_{24,2}=\left\{\left(s_{5}, 0.4\right)\right. \\
\left.\left(s_{6}, 0.6\right)\right\}\end{array}$ \\
\hline$A_{3}$ & $\begin{array}{c}m_{31,2}=\left\{\left(s_{4}, 0.4\right)\right. \\
\left.\left(s_{5}, 0.6\right)\right\}\end{array}$ & $\begin{array}{l}m_{32,2}=\left\{\left(s_{1}, 0.2\right)\right. \\
\left.\left(s_{2}, 0.2\right),\left(s_{3}, 0.6\right)\right\}\end{array}$ & $\begin{array}{l}m_{33,2}=\left\{\left(s_{2}, 0.3\right)\right. \\
\left.\left(s_{3}, 0.4\right),\left(s_{4}, 0.3\right)\right\}\end{array}$ & $\begin{array}{l}m_{34,2}=\left\{\left(s_{4}, 0.2\right),\right. \\
\left.\left(s_{5}, 0.6\right),\left(s_{6}, 0.2\right)\right\}\end{array}$ \\
\hline$A_{4}$ & $\begin{array}{c}m_{41,2}=\left\{\left(s_{2}, 0.5\right)\right. \\
\left.\left(s_{3}, 0.4\right),\left(s_{4}, 0.1\right)\right\}\end{array}$ & $\begin{array}{l}m_{42,2}=\left\{\left(s_{3}, 0.3\right)\right. \\
\left.\left(s_{4}, 0.5\right),\left(s_{5}, 0.2\right)\right\}\end{array}$ & $\begin{array}{l}m_{43,2}=\left\{\left(s_{4}, 0.1\right)\right. \\
\left.\left(s_{5}, 0.2\right),\left(s_{6}, 0.7\right)\right\}\end{array}$ & $\begin{array}{l}m_{44,2}=\left\{\left(s_{4}, 0.3\right),\right. \\
\left.\left(s_{5}, 0.5\right),\left(s_{6}, 0.2\right)\right\}\end{array}$ \\
\hline
\end{tabular}

Table 4 The individual linguistic distribution assessments matrix $M_{3}$.

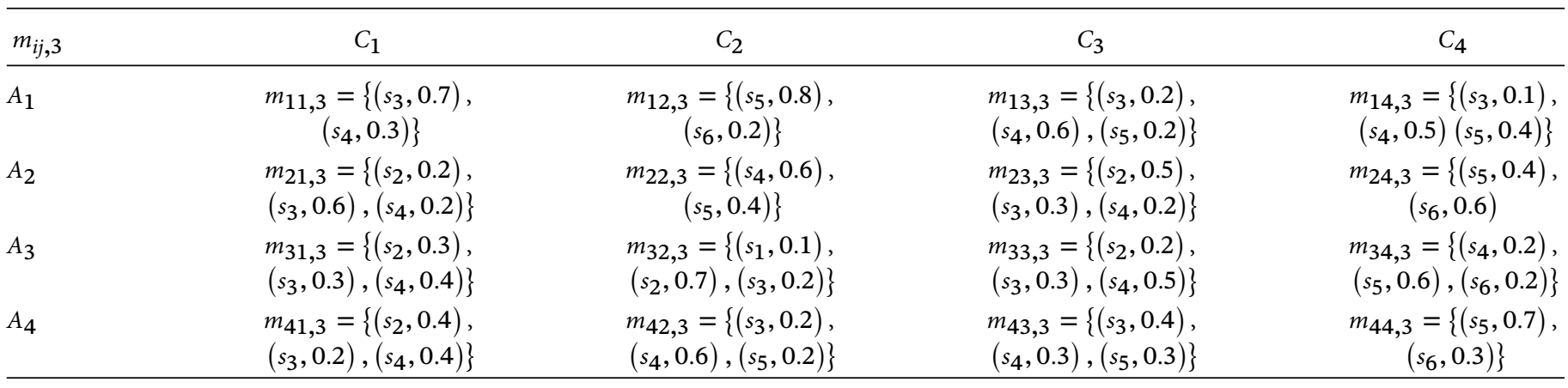

Table 5 The collective decision matrix $M_{c o l}^{(0)}$.

\begin{tabular}{|c|c|c|c|c|}
\hline$m_{i j, c o l}$ & $C_{1}$ & $C_{2}$ & $C_{3}$ & $C_{4}$ \\
\hline$A_{1}$ & $\begin{array}{l}m_{11, c o l}=\left\{\left(s_{2}, 0.17\right)\right. \\
\left.\left(s_{3}, 0.50\right),\left(s_{4}, 0.33\right)\right\}\end{array}$ & $\begin{array}{l}m_{12, c o l}=\left\{\left(s_{4}, 0.20\right)\right. \\
\left.\left(s_{5}, 0.67\right),\left(s_{6}, 0.13\right)\right\}\end{array}$ & $\begin{array}{l}m_{13, c o l}=\left\{\left(s_{3}, 0.27\right)\right. \\
\left.\left(s_{4}, 0.43\right),\left(s_{5}, 0.30\right)\right\}\end{array}$ & $\begin{array}{c}m_{14, c o l}=\left\{\left(s_{2}, 0.17\right),\right. \\
\left(s_{3}, 0.20\right),\left(s_{4}, 0.43\right), \\
\left.\left(s_{5}, 0.20\right)\right\}\end{array}$ \\
\hline$A_{2}$ & $\begin{array}{c}m_{21, c o l}=\left\{\left(s_{2}, 0.10\right),\right. \\
\left(s_{3}, 0.53\right),\left(s_{4}, 0.30\right), \\
\left.\left(s_{5}, 0.07\right)\right\}\end{array}$ & $\begin{array}{l}m_{22, c o l}=\left\{\left(s_{3}, 0.17\right)\right. \\
\left.\left(s_{4}, 0.50\right),\left(s_{5}, 0.33\right)\right\}\end{array}$ & $\begin{array}{c}m_{23, c o l}=\left\{\left(s_{2}, 0.30\right),\right. \\
\left(s_{3}, 0.37\right),\left(s_{4}, 0.27\right), \\
\left.\left(s_{5}, 0.06\right)\right\}\end{array}$ & $\begin{array}{c}m_{24, c o l}=\left\{\left(s_{5}, 0.50\right)\right. \\
\left.\left(s_{6}, 0.50\right)\right\}\end{array}$ \\
\hline$A_{3}$ & $\begin{array}{c}m_{31, c o l}=\left\{\left(s_{2}, 0.10\right),\right. \\
\left(s_{3}, 0.10\right),\left(s_{4}, 0.37\right), \\
\left.\left(s_{5}, 0.43\right)\right\}\end{array}$ & $\begin{array}{l}m_{32, c o l}=\left\{\left(s_{1}, 0.20\right)\right. \\
\left.\left(s_{2}, 0.50\right),\left(s_{3}, 0.30\right)\right\}\end{array}$ & $\begin{array}{l}m_{33, c o l}=\left\{\left(s_{2}, 0.33\right)\right. \\
\left.\left(s_{3}, 0.37\right),\left(s_{4}, 0.30\right)\right\}\end{array}$ & $\begin{array}{l}m_{34, c o l}=\left\{\left(s_{4}, 0.13\right)\right. \\
\left.\left(s_{5}, 0.60\right),\left(s_{6}, 0.27\right)\right\}\end{array}$ \\
\hline$A_{4}$ & $\begin{array}{l}m_{41, c o l}=\left\{\left(s_{2}, 0.37\right)\right. \\
\left.\left(s_{3}, 0.33\right),\left(s_{4}, 0.30\right)\right\}\end{array}$ & $\begin{array}{c}m_{42, c o l}=\left\{\left(s_{3}, 0.17\right)\right. \\
\left(s_{4}, 0.57\right),\left(s_{5}, 0.20\right) \\
\left.\left(s_{6}, 0.06\right)\right\}\end{array}$ & $\begin{array}{l}m_{43, c o l}=\left\{\left(s_{3}, 0.30\right)\right. \\
\left.\left(s_{4}, 0.30\right),\left(s_{5}, 0.40\right)\right\}\end{array}$ & $\begin{array}{l}m_{44, c o l}=\left\{\left(s_{4}, 0.20\right)\right. \\
\left.\left(s_{5}, 0.63\right),\left(s_{6}, 0.17\right)\right\}\end{array}$ \\
\hline
\end{tabular}


Step 7. Elicit preference information about $d_{31, t}^{k,-}$ and $d_{31, t}^{k,+}, t=$ 1,$3 ; k=0,1, \ldots, 6$. In order to support experts $e_{1}$ and $e_{3}$ to provide additional preference information, the following vectors determined by Equations (19) and (20) can be provided to them as a reference:

$$
\begin{aligned}
& B_{31,1}^{-,(0)}=(0.00,0.00,0.00,0.00,0.00,0.27,0.00,) \\
& B_{31,1}^{+,(0)}=(0.00,0.00,0.10,0.10,0.07,0.00,0.00,) \\
& B_{31,3}^{-,(0)}=(0.00,0.00,0.20,0.20,0.03,0.00,0.00,) \\
& B_{31,3}^{+,(0)}=(0.00,0.00,0.00,0.00,0.00,0.43,0.00 .)
\end{aligned}
$$

Suppose that experts $e_{1}$ and $e_{3}$ provide the following additional preferences:

$$
\begin{aligned}
& d_{31,1}^{L B}=(0.00,0.00,0.00,0.00,0.00,0.20,0.00,) \\
& d_{31,1}^{U B}=(0.00,0.00,0.10,0.10,0.00,0.00,0.00,) \\
& d_{31,3}^{L B}=(0.00,0.00,0.10,0.20,0.00,0.00,0.00,) \\
& d_{31,3}^{U B}=(0.00,0.00,0.00,0.00,0.00,0.20,0.00 .)
\end{aligned}
$$

Step 8. Based on the preference information provided by experts $e_{1}$ and $e_{3}$, the optimization model $(M-3)$ can be constructed. By solving the optimization model, we obtain the following nonzero components of the optimal solution: $d_{31,3}^{3,-}=0.0838, d_{31,3}^{5,+}=0.0838$.

Step 9. Adjust the linguistic distribution assessments. According to the optimal solution of the model $(M-3)$, we can see that only the expert $e_{3}$ need to adjust his preference in position $(3,1)$. The adjusted preference is $m_{31,3}^{\prime}=\left\{\left(s_{0}, 0\right),\left(s_{1}, 0\right),\left(s_{2}, 0.3\right),\left(s_{3}, 0.22\right),\left(s_{4}, 0.4\right),\left(s_{5}, 0.08\right)\right.$, $\left.\left(s_{6}, 0\right)\right\}$.

Step 10. Determine the updated consensus matrix. Based on the modified decision matrices, we can calculate the updated consensus matrix $S_{(1)}^{c o l}$ as follows:

$$
S_{(1)}^{c o l}=\left(\begin{array}{llll}
0.9741 & 0.9630 & 0.9815 & \mathbf{0 . 8 9 2 6} \\
0.9556 & 0.9556 & 0.9111 & 0.9778 \\
0.8976 & 0.9556 & 0.9593 & 0.9704 \\
0.9563 & 0.9519 & 0.9444 & 0.9593
\end{array}\right)
$$

We can see that $G C I=0.8926<\overline{C I}$. Thus, the iteration should continue.

In this example, the predefined consensus level is achieved after six rounds of iterations. Compared with the original decision matrices, the expert $e_{1}$ does not adjust his preferences. The expert $e_{2}$ updates the preference $m_{14,2}$ and $m_{23,2}$ to $m_{14,2}^{\prime}=\left\{\left(s_{2}, 0.42\right),\left(s_{3}, 0.17\right),\left(s_{4}, 0.27\right),\left(s_{5}, 0.14\right)\right\}$ and $m_{23,2}^{\prime}=\left\{\left(s_{2}, 0.04\right),\left(s_{3}, 0.30\right),\left(s_{4}, 0.50\right),\left(s_{5}, 0.16\right)\right\}$, respectively. The expert $e_{3}$ updates the preference $m_{31,3}$ to $m_{31,3}^{\prime}=$ $\left\{\left(s_{2}, 0.24\right),\left(s_{3}, 0.15\right),\left(s_{4}, 0.40\right),\left(s_{5}, 0.21\right)\right\}$. The final collective decision matrix are show in Table 6.
In the following, the selection process of the GDM model is implemented.

Based on the final collective decision matrix $M_{c o l}$, we can obtain the following overall values of each alternative by using the known weights of attributes and $D A W A_{W}$ operator: $m_{1, c o l}=$ $\left\{\left(s_{2}, 0.08\right),\left(s_{3}, 0.25\right),\left(s_{4}, 0.35\right),\left(s_{5}, 0.29\right),\left(s_{6}, 0.03\right)\right\}, m_{2, c o l}=$ $\left\{\left(s_{2}, 0.11\right),\left(s_{3}, 0.29\right),\left(s_{4}, 0.28\right),\left(s_{5}, 0.22\right),\left(s_{6}, 0.10\right)\right\}, m_{3, \text { col }}=$ $\left\{\left(s_{1}, 0.05\right),\left(s_{2}, 0.23\right),\left(s_{3}, 0.18\right),\left(s_{4}, 0.21\right),\left(s_{5}, 0.27\right),\left(s_{6}, 0.06\right)\right\}$, $m_{4, c o l}=\left\{\left(s_{2}, 0.11\right),\left(s_{3}, 0.22\right),\left(s_{4}, 0.35\right),\left(s_{5}, 0.28\right),\left(s_{6}, 0.04\right)\right\}$,

By using Eq. (2), we can further obtain the expectation of each alternative, i.e., $E\left(m_{1, c o l}\right)=\left(s_{4},-0.0455\right), E\left(m_{2, c o l}\right)=\left(s_{4},-0.0933\right)$, $E\left(m_{3, c o l}\right)=\left(s_{4},-0.4197\right), E\left(m_{4, c o l}\right)=\left(s_{4},-0.0600\right)$. According to the values of $E\left(m_{i, c o l}\right), i=1,2,3,4$, the ranking of four alternatives is $x_{1}>x_{4}>x_{2}>x_{3}$.

\subsection{Comparison Analysis}

In order to demonstrate the differences between the proposed method and other relevant methods and emphasize the advantages and characteristics of the proposed method, in this section we conduct comparison analysis to evaluate the performance of the proposed consensus reaching model.

Regarding MAGDM problem with linguistic distribution assessments, a consensus reaching model is proposed in this paper to help decision makers to achieve agreement in decision-making. In order to analyze the impact of CRP on decision-making, by using the numerical example in Section 5, we compare the result calculated by our model with that calculated by the MAGDM model without CRP. In the numerical example, we can aggregate the original individual decision matrices by using the $D A W A_{W}$ operator. Further, based on the collective decision matrix, that is, $M_{c o l}^{(0)}$, the following expectation of each alternative can be obtained by using the known weights of attributes and $D A W A_{W}$ operator: $E\left(m_{1, c o l}\right)=\left(s_{4},-0.0750\right), E\left(m_{2, c o l}\right)=\left(s_{4},-0.0833\right), E\left(m_{3, c o l}\right)=$ $\left(s_{4},-0.4667\right), E\left(m_{4, c o l}\right)=\left(s_{4},-0.0600\right)$. According to the expectation values, the ranking of four alternatives is $x_{4}>x_{1}>x_{2}>x_{3}$. We can see that CRP has a certain effect on the ranking results in this example. However, it is pointed out that the ranking results based on the proposed model have better consensus, which is very important in practice.

In [7], Zhang et al. developed a consensus model for GDM with linguistic distribution assessments based on the IR and the adjustment rule. Here, we compare the consensus model in [7] and the proposed consensus model in the present paper from the following aspects. In the first place, although both models are concerned with consensus issue in GDM problems with linguistic distribution assessments, the proposed consensus model is developed for GDM problems in which the decision information is represented by means of multi-attribute decision matrix, while the consensus model in [7] is proposed for GDM problem with PRs. Secondly, in the consensus model in [7], an automatic feedback strategy is adopted by using the weighted average of the distribution linguistic PRs provided by all the experts as the adjusted distribution linguistic preference. One advantage of this strategy is that it is easy to generate adjustment advices. However, the preferences of the identified expert can not be taken into account in the CRP. In contrast, the proposed model allows experts who are identified to 
Table 6 The final collective decision matrix $M_{c o l}$.

\begin{tabular}{|c|c|c|c|c|}
\hline$x_{i j}$ & $C_{1}$ & $C_{2}$ & $C_{3}$ & $C_{4}$ \\
\hline$A_{1}$ & $\begin{array}{l}m_{11, c o l}=\left\{\left(s_{2}, 0.17\right)\right. \\
\left.\left(s_{3}, 0.50\right),\left(s_{4}, 0.33\right)\right\}\end{array}$ & $\begin{array}{l}m_{12, c o l}=\left\{\left(s_{4}, 0.20\right),\right. \\
\left.\left(s_{5}, 0.67\right),\left(s_{6}, 0.13\right)\right\}\end{array}$ & $\begin{array}{l}m_{13, c o l}=\left\{\left(s_{3}, 0.27\right)\right. \\
\left.\left(s_{4}, 0.43\right),\left(s_{5}, 0.30\right)\right\}\end{array}$ & $\begin{array}{c}m_{14, c o l}=\left\{\left(s_{2}, 0.14\right),\right. \\
\left(s_{3}, 0.16\right),\left(s_{4}, 0.45\right), \\
\left.\left(s_{5}, 0.25\right)\right\}\end{array}$ \\
\hline$A_{2}$ & $\begin{array}{c}m_{21, c o l}=\left\{\left(s_{2}, 0.10\right)\right. \\
\left(s_{3}, 0.53\right),\left(s_{4}, 0.30\right) \\
\left.\left(s_{5}, 0.07\right)\right\}\end{array}$ & $\begin{array}{l}m_{22, c o l}=\left\{\left(s_{3}, 0.20\right)\right. \\
\left.\left(s_{4}, 0.60\right),\left(s_{5}, 0.20\right)\right\}\end{array}$ & $\begin{array}{c}m_{23, c o l}=\left\{\left(s_{2}, 0.31\right)\right. \\
\left(s_{3}, 0.37\right),\left(s_{4}, 0.27\right) \\
\left.\left(s_{5}, 0.05\right)\right\}\end{array}$ & $\begin{array}{c}m_{24, c o l}=\left\{\left(s_{5}, 0.50\right)\right. \\
\left.\left(s_{6}, 0.50\right)\right\}\end{array}$ \\
\hline$A_{3}$ & $\begin{array}{c}m_{31, c o l}=\left\{\left(s_{2}, 0.08\right),\right. \\
\left(s_{3}, 0.05\right),\left(s_{4}, 0.37\right) \\
\left.\left(s_{5}, 0.50\right)\right\}\end{array}$ & $\begin{array}{l}m_{32, \text { col }}=\left\{\left(s_{1}, 0.20\right)\right. \\
\left.\left(s_{2}, 0.50\right),\left(s_{3}, 0.30\right)\right\}\end{array}$ & $\begin{array}{l}m_{33, c o l}=\left\{\left(s_{2}, 0.33\right)\right. \\
\left.\left(s_{3}, 0.37\right),\left(s_{4}, 0.30\right)\right\}\end{array}$ & $\begin{array}{l}m_{34, c o l}=\left\{\left(s_{4}, 0.13\right),\right. \\
\left.\left(s_{5}, 0.60\right),\left(s_{6}, 0.27\right)\right\}\end{array}$ \\
\hline$A_{4}$ & $\begin{array}{l}m_{41, c o l}=\left\{\left(s_{2}, 0.33\right),\right. \\
\left.\left(s_{3}, 0.33\right),\left(s_{4}, 0.34\right)\right\}\end{array}$ & $\begin{array}{c}m_{42, c o l}=\left\{\left(s_{3}, 0.17\right)\right. \\
\left(s_{4}, 0.57\right),\left(s_{5}, 0.20\right) \\
\left.\left(s_{6}, 0.06\right)\right\}\end{array}$ & $\begin{array}{c}m_{43, c o l}=\left\{\left(s_{3}, 0.23\right)\right. \\
\left(s_{4}, 0.37\right),\left(s_{5}, 0.33\right) \\
\left.\left(s_{6}, 0.07\right)\right\}\end{array}$ & $\begin{array}{l}m_{44, c o l}=\left\{\left(s_{4}, 0.20\right),\right. \\
\left.\left(s_{5}, 0.63\right),\left(s_{6}, 0.17\right)\right\}\end{array}$ \\
\hline
\end{tabular}

modify their preferences to provide additional opinions in each iteration of the CRP. Finally, the feedback mechanism in the proposed consensus model considers the preservation of the initial preferences of the experts, which is not considered in the consensus model proposed by Zhang et al.

\section{CONCLUSIONS}

The CRP dedicated to obtaining a maximum degree of agreement between a set of decision makers is an important aspect in MAGDM problems. This study proposes a novel consensus model for MAGDM problem with information represented by means of linguistic distribution assessments. Compared with the classical MAGDM models, the proposed method has the following characteristics: (1) In order to develop the consensus reaching model for MAGDM problem with linguistic distribution assessments, a novel distance measure between linguistic distribution assessments is proposed to overcome the limitations of the existing distance measure. (2)The proposed consensus reaching model for MAGDM problem with linguistic distribution assessments can not only reflect the experts additional opinions during the CRP, but also automatically generate advices for preference adjustment. (3) In the feedback mechanism of the proposed model, an optimization model is solved in each iteration to minimize the deviation between the adjusted values and initial preferences, which in turn leads to the good performance of the proposed consensus reaching model in preserving the initial preference information.

Although experts only need to provide the proportional distribution information on HFLTS with a few linguistic terms in the proposed decision model, they may have cognitive difficulties in understanding the adjusted general linguistic distribution assessments outputted by the CRP algorithm. It would be better to represent the adjusted preferences in the form of linguistic distribution assessments with no more than three linguistic terms, which is an issue worthy of further investigation. In real-world MAGDM problems, the set of alternatives and the participation of decision makers may change dynamically. Therefore, it will be very interesting in future research to incorporate the dynamic changes of alternatives and decision makers into the developed consensus reaching model. Meanwhile, the expansion of technological paradigms call for the public attention for the large-scale MAGDM (LMAGDM) problems, in which a larger number of experts take part in the decision process and responsibility for the decision result. We point out that it will be interesting to investigate LMAGDM with linguistic distribution assessments in the consensus building.

\section{ACKNOWLEDGMENTS}

The authors are very grateful to the editor and the anonymous reviewers for their insightful and constructive comments and suggestions that have led to an improved version of this paper. This work is partially supported by the Soft Science Research Projects of Technical Innovation in Hubei Province in 2018 (Grant no. 2018ADC085).

\section{REFERENCES}

[1] D.M. Carlos. A multicriteria decision analysis model for faculty evaluation, Omega. 40 (2012), 424-436.

[2] D. Hochbaum, A. Levin, Methodologies and algorithms for group-rankings decision, Manag. Sci. 52 (2006), 1394-1408.

[3] J.M. Mendel, Computing with words and its relationships with fuzzistics, Info. Sci. 4 (2007), 988-1006.

[4] F. Herrera, L. Martínez, A 2-tuple fuzzy linguistic representation model for computing with words, IEEE Trans. Fuzzy Syst. 8(6) (2000), 746-752.

[5] R.M. Rodríguez, L. Martinez, F. Herrera, Hesitant fuzzy linguistic term sets for decision making, IEEE Trans. Fuzzy Syst. 20(1) (2012), 109-119.

[6] R.M. Rodríguez, Á. Labella, L. Martínez, An overview on fuzzy modelling of complex linguistic preferences in decision making, Int. J. Comput. Intell. Syst. 9 (2016), 81-94.

[7] G.Q. Zhang, Y.C. Dong, Y.F. Xu, Consistency and consensus measures for linguistic preference relations based on distribution assessments, Info. Fusion, 17 (2014), 46-55.

[8] Z.B. Wu, J. Xu, Possibility distribution-based approach for magdm with hesitant fuzzy linguistic information, IEEE Trans. Cybern. 46 (2016), 694-705.

[9] Z.S. Chen, K. Chin, Y. Li, Y. Yang, Proportional hesitant fuzzy linguistic term set for multiple criteria group decision making, Info. Sci. 357 (2016), 61-87. 
[10] Q. Pang, H. Wang, Z.S. Xu, Probabilistic linguistic term sets in multi-attribute group decision making, Info. Sci. 369 (2016), $128-143$.

[11] H.C. Liao, L.S. Jiang, Z.S. Xu, A linear programming method for multiple criteria decision making with probabilistic linguistic information, Info. Sci. 415 (2017), 341-355.

[12] H. Yan, T. Ma, V.-N. Huynh, On qualitative multi-attribute group decision making and its consensus measure: a probability based perspective, Omega. 70 (2017), 94-117.

[13] Y. Dong, Y. Wu, H. Zhang, G. Zhang, Multi-granular unbalanced linguistic distribution assessments with interval symbolic proportions, Knowl.-Based Syst. 82 (2015), 139-151.

[14] Z. Zhang, C. Guo, L. Martínez, Managing multigranular linguistic distribution assessments in large-scale multiattribute group decision making, IEEE Trans. Syst. Man Cybern. Syst. 47 (2017), 3063-3076.

[15] S. Yu, J. Wang, J. Wang, L. Li, A multi-criteria decision-making model for hotel selection with linguistic distribution assessments, Appl. Soft Comput. 67 (2018), 741-755.

[16] J. Kacprzyk, M. Fedrizzi, A soft measure of consensus in the setting of partial (fuzzy) preferences, Eur. J. Oper. Res. 34 (1988), 316-323.

[17] J. Cabrerizo, F.J. Moreno, I. prez, E. Herrera-Viedma, Analyzing consensus approaches in fuzzy group decision making: advantages and drawbacks, Soft Comput. 14 (2010), 451-463.

[18] E. Herrera-Viedma, L. Martinez, F. Mata, F. Chiclana, A consensus support system model for group decision-making problems with multigranular linguistic preference relations, IEEE Trans. Fuzzy Syst. 13(5) (2005), 644-658.

[19] Y.C. Dong, G.Q. Zhang, W.C. Hong, Consensus models for ahp group decision making under row geometric mean prioritization method, Decis. Support Syst. 49 (2010), 281-289.

[20] J. Garcia, M. Del Moral, M. Martinez, E. Herrera-Viedma, A consensus model for group decision-making problems with interval fuzzy preference relations, Int. J. Info. Technol. Decis.-Mak. 11 (2012), 709-725.

[21] Y.J. Xu, C.Y. Li, X.W. Wen, Missing values estimation and consensus building for incomplete hesitant fuzzy preference relations with multiplicative consistency, Int. J. Comput. Intell. Syst. 11 (2018), 101-119.

[22] Á. Labella, Y. Liu, R.M. Rodríguez, L. Martínez, Analyzing the performance of classical consensus models in large scale group decision making: a comparative study, Appl. Soft Comput. 67 (2018), 677-690.

[23] R.M. Rodríguez, Á. Labella, G.D. Tré, L. Martínez, A large scale consensus reaching process managing group hesitation, Knowl.Based Syst. 159 (2018), 86-97.

[24] D. Ben-Arieh, Z. Chen, Linguistic-labels aggregation and consensus measure for autocratic decision making using group recommendations, IEEE Trans. Syst. Man Cyber. Part A Syst. Hum. 36 (2006), 558-568.

[25] F. Mata, L. Martinez, E. Herrera-Viedma, An adaptive consensus support model for group decision-making problems in a multigranular fuzzy linguistic context, IEEE Trans. Fuzzy Syst. 17 (2009), 279-290.
[26] F.J. Cabrerizo, S. Alonso, E. Herrera-Viedma, A consensus model for group decision making problems with unbalanced fuzzy linguistic information, Int. J. Info. Technol. Decis. Mak. 8(01) (2009), 109-131.

[27] S. Alonso, E. Herrera-Viedma, F. Chiclana, F. Herrera, A web based consensus support system for group decision making problems and incomplete preferences, Info. Sci. 180(23) (2010), 4477-4495.

[28] Y. Dong, Y. Xu, H. Li, B. Feng, The owa-based consensus operator under linguistic representation models using position indexes, Eur. J. Oper. Res. 203(2) (2010), 455-463.

[29] J. Garcia, M. Del Moral, M. Martinez, E. Herrera-Viedma, A consensus model for group decision making problems with linguistic interval fuzzy preference relations, Expert Syst. Appl. 39 (2012), 10022-10030.

[30] Z. Wu, J. Xu, Consensus reaching models of linguistic preference relations based on distance functions, Soft Comput. 16(4) (2012), 577-589.

[31] S. Alonso, I.J. Pérez, F.J. Cabrerizo, E. Herrera-Viedma, A linguistic consensus model for web 2.0 communities, Appl. Soft Comput. 13(1) (2013), 149-157.

[32] Z.W. Gong, J. Forrest, Y.-J. Yang, The optimal group consensus models for 2-tuple linguistic preference relations, Knowl.-Based Syst. 37 (2013), 427-437.

[33] Y.C. Dong, Z.P. Fan, S. Yu, Consensus building in a local context for the ahp-gdm with the individual numerical scale and prioritization method, IEEE Trans. Fuzzy Syst. 23 (2015), 354-368.

[34] Y. Xu, S.H., H. Wang, Optimal consensus models for group decisionmaking under linguistic preference relations, Int. Trans. Oper. Res. 23 (2016), 1201-1228.

[35] Z. Zhang, C. Guo, Consistency and consensus models for group decision-making with uncertain 2-tuple linguistic preference relations, Int. J. Syst. Sci. 47(11) (2016), 2572-2587.

[36] C. Li, Y.C. Dong, F. Herrera, E. Herrera-Viedma, L. Martinez, Personalized individual semantics in computing with words for supporting linguistic group decision making, Info. Fusion. 33 (2017), 29-44.

[37] Y.J. Xu, X.W. Wen, H. Sun, Consistency and consensus models with local adjustment strategy for hesitant fuzzy linguistic preference relations, Int. J. Fuzzy Syst. 20 (2018), 2216-2233.

[38] Z.S. Xu, F. Herrera, An interactive approach to multiple attribute group decision making with multi granular uncertain linguistic information, Group Decis. Negot. 18 (2009), 119-145.

[39] R.O. Parreiras, P.Y. Ekel, J.S.C. Martini, A flexible consensus scheme for multicriteria group decision making under linguistic assessments, Info. Sci. 180 (2010), 1075-1089.

[40] L. Rosello, M. Sanchez, N. Agell, Using consensus and distances between generalized multi-attribute linguistic assessments for group decision-making, Info. Fusion. S1 (2014), 83-92.

[41] J.P. Xu, Z.B. Wu, Y. Zhang, A consensus based method for multicriteria group decision making under uncertain linguistic setting, Group Decis. Negot. 23 (2014), 127-148.

[42] J. Wu, F. Chiclana, E. Herrera-Viedma, Trust based consensus model for social network in an incomplete linguistic information context, Appl. Soft Comput. 35 (2015), 827-839.

[43] Z. Sun, W.M. Ma, An approach to consensus measurement of linguistic preference relations in multi-attribute group decision making and application, OMEGA. 51 (2015), 83-92. 
[44] W.C. Zhang, Y.J. Xu, H.M. Wang, A consensus reaching model for 2-tuple linguistic multiple attribute group decision making with incomplete weight information, Int. J. Syst. Sci. 47 (2016), 389-405.

[45] J.F. Pang, J.Y. Liang, P. Song, An adaptive consensus method for multi-attribute group decision making under uncertain linguistic environment, Appl. Soft Comput. 58 (2017), 339-353.

[46] B.W. Zhang, H.M. Liang, G.Q. Zhang, Reaching a consensus with minimum adjustment in magdm with hesitant fuzzy linguistic term sets, Info. Fusion. 42 (2018), 12-23.
[47] X.Y. Zhang, J.Q. Wang, J.H. Hu, A consensus approach to multi-granular linguistic mcgdm with hesitant fuzzy linguistic information by using projection, J. Intell. Fuzzy Syst. 34 (2018), 1959-1974.

[48] H.C. Liao, Z.S. Xu, X.J. Zeng, Distance and similarity measures for hesitant fuzzy linguistic term sets and their application in multicriteria decision making, Info. Sci. 271 (2014), 125-142. 